\title{
FORCING CONSEQUENCES OF PFA TOGETHER WITH THE CONTINUUM LARGE
}

\author{
DAVID ASPERÓ AND MIGUEL ANGEL MOTA
}

\begin{abstract}
We develop a new method for building forcing iterations with symmetric systems of structures as side conditions. Using this method we prove that the forcing axiom for the class of all finitely proper posets of size $\aleph_{1}$ is compatible with $2^{\aleph_{0}}>\aleph_{2}$. In particular, this answers a question of Moore by showing that $\mho$ does not follow from this arithmetical assumption.
\end{abstract}

\section{INTRODUCTION}

In the early days of forcing, Solovay and Tennenbaum (see 22]) developed the theory of c.c.c. iterations in order to show the consistency of Suslin's Hypothesis (i.e., the axiom saying that there are no Suslin trees). In fact, as they realised, their technique could be used to build models of ZFC with the continuum arbitrarily large and satisfying a condition much stronger - in the presence of $\neg \mathrm{CH}$ - than Suslin's Hypothesis that came to be known as Martin's Axiom. Recall that a partial order has the countable chain condition (c.c.c.) if it has no uncountable antichains. Given a cardinal $\kappa$, Martin's Axiom for $\kappa$-many dense sets, $\mathrm{MA}_{\kappa}$, is the forcing axiom for the class of c.c.c. forcing notions and for collections of $\kappa$-many dense sets, i.e. the axiom saying that for any c.c.c. partial order $\mathbb{P}$ and any collection $\mathcal{D}$ of $\kappa$-many dense subsets of $\mathbb{P}$ there is a filter $G \subseteq \mathbb{P}$ having nonempty intersection with all members of $\mathcal{D}$, and Martin's Axiom is $\mathrm{MA}_{\kappa}$ for all $\kappa<2^{\aleph_{0}}$. Martin's Axiom (typically in the form $\mathrm{MA}_{\omega_{1}}$ ) proved to be very successful in applications in infinite combinatorics, topology, algebra, and other areas of mathematics (see [7]). The main features of c.c.c. forcing are that (1) c.c.c. forcing notions preserve all cardinals, and (2) finite support iterations of c.c.c. forcing notions are themselves c.c.c. It follows from these two facts together that no forcing axiom of the form $\mathrm{MA}_{\kappa}$ puts any upper bound on the size of the continuum (on the other hand, $\mathrm{MA}_{\kappa}$ certainly implies $2^{\aleph_{0}}>\kappa$ ).

About a decade later, the theory of proper forcing was developed by Shelah ([19]; see also [20], [21]). A poset $\mathbb{P}$ is proper if for every regular cardinal $\lambda>|T C(\mathbb{P})|$, every countable $N \preccurlyeq H(\lambda)$ such that $\mathbb{P} \in N$, and every $p \in \mathbb{P} \cap N$ there is a condition $q$ in $\mathbb{P}$ stronger than $p$ which is $(N, \mathbb{P})$-generic, i.e., such that $q$ forces $\dot{G} \cap D \cap N \neq \emptyset$ for every dense subset $D$ (equivalently, maximal antichain) of $\mathbb{P}$ such that $D \in N$, where $\dot{G}$ is the canonical name for the generic filter. The class of proper forcings is much larger than the class of c.c.c. forcings. Nevertheless, proper forcings are well

Received by the editors October 4, 2011 and, in revised form, May 31, 2013.

2010 Mathematics Subject Classification. Primary 03E50, 03E57, 03E35, 03 E05.

The second author was supported by the Austrian Science Fund FWF Project P22430. Both authors were also partially supported by Ministerio de Educación y Ciencia Project MTM200803389 (Spain) and by Generalitat de Catalunya Project 2009SGR-00187 (Catalonia). 
behaved in the sense that (1) they preserve $\omega_{1}$, and that (2) every countable support iteration with proper iterands is itself proper. Baumgartner showed the consistency of the forcing axiom for the class of proper forcings and for collections of $\aleph_{1}$-many dense sets, also known as the Proper Forcing Axiom (PFA), by building countable support iterations of proper forcing notions of length a supercompact cardinal (see [3]). Soon it was realised that PFA has many consequences (see for example [3] for a classic overview). One remarkable consequence of PFA (by Todorčević and Veličković; see [4] and [25]) is that, unlike any forcing axiom of the form $\mathrm{MA}_{\kappa}$, it does decide the value of $2^{\aleph_{0}}$; in fact, PFA implies $2^{\aleph_{0}}=\aleph_{2} 11$ More recently, Moore has proved (see [14]) that $2^{\aleph_{0}}=\aleph_{2}$ already follows from the bounded form of PFA known as BPFA (BPFA can be phrased as the axiom saying that $\left\langle H\left(\omega_{2}\right), \in\right\rangle$ is a $\Sigma_{1}$ elementary substructure of the structure $\left\langle H\left(\omega_{2}\right), \in\right\rangle$ as computed in any generic extension by a proper forcing).

Given that strong forcing axioms typically imply $2^{\aleph_{0}}=\aleph_{2}$, a natural problem when faced with a consequence $\sigma$ of a forcing axiom is to find out whether $\sigma$ itself has any impact on the size of the continuum and which. The standard strategy for producing models of $\Pi_{2}$ consequences $\sigma$ (over the structure $\left\langle H\left(\omega_{2}\right), \in, \omega_{1}\right\rangle$ ) of forcing axioms is by means of forcing iterations in which one keeps adding witnesses of the relevant $\Sigma_{1}$ facts 2 If it can be shown that there is always a forcing adding these witnesses which moreover has the c.c.c., then a sufficiently long finite support iteration of (carefully chosen) instances of this forcing will produce a model of $\sigma$. Since finite support iterations of c.c.c. forcings are themselves c.c.c. and since c.c.c. forcings preserve cardinals, such a construction will give rise to models of $\sigma$ in which $2^{\aleph_{0}}$ can attain (almost) any arbitrarily fixed value. For example, this is the standard way of showing that Suslin's Hypothesis is consistent with $2^{\aleph_{0}}$ being any arbitrarily fixed $\aleph_{\alpha}$ with $c f\left(\aleph_{\alpha}\right)>\omega_{1}$ (see 22] 3 In fact, if GCH holds and $\kappa$ is any cardinal of uncountable cofinality, then a certain finite support iteration of length $\kappa$ will produce a model of $2^{\aleph_{0}}=\kappa$ together with $\mathrm{MA}_{\lambda}$ for all $\lambda<c f(\kappa)$, and already $\mathrm{MA}_{\omega_{1}}$ implies Suslin's Hypothesis (22]).

However, one often deals with statements $\sigma$ that cannot be changed by c.c.c. forcing. Consider for example Club Guessing (CG), which says that there is a ladder system on $\omega_{1}$ (a ladder system is a sequence $\left\langle A_{\delta}: \delta \in \operatorname{Lim}\left(\omega_{1}\right)\right\rangle$ such that each $A_{\delta}$ is a cofinal subset of $\delta$ of order type $\omega$ ) that 'guesses' clubs, in the sense that if $C \subseteq \omega_{1}$ is a club, then there is some limit ordinal $\delta<\omega_{1}$ such that a final segment of $A_{\delta}$ is contained in $C$. Club Guessing is clearly a consequence of Jensen's $\diamond$. However, unlike $\diamond$, Club Guessing is immune to c.c.c. forcing. In fact, every club of $\omega_{1}$ in any generic extension by a c.c.c. forcing contains a club from the ground model, and therefore Club Guessing holds in any extension by any c.c.c. forcing if it happens to hold in the ground model. Now take our statement

\footnotetext{
${ }^{1}$ The first derivation of $2^{\aleph_{0}}=\aleph_{2}$ from a natural forcing axiom was the proof, due to Foreman, Magidor and Shelah (see [6]), that Martin's Maximum, which is a provably maximal forcing axiom for collections of $\aleph_{1}$-many dense sets and is strictly stronger than PFA, implies $2^{\aleph_{0}}=\aleph_{2}$.

${ }^{2}$ This is also the traditional way of building models of actual forcing axioms like $\mathrm{MA}_{\kappa}, \mathrm{BPFA}$, PFA, or Martin's Maximum.

${ }^{3}$ As proved by Jensen ([5]), Suslin's hypothesis is also consistent with $\mathrm{CH}$, but the proof of this uses a countable - rather than finite - support iteration. Shelah's 21] is a classical reference on forcing $\Pi_{2}$ statements over $\left\langle H\left(\omega_{2}\right), \in, \omega_{1}\right\rangle$ together with $\mathrm{CH}$ by means of countable support iterations. Also, Laver proved in 12 that adding any number of random reals to any model of $\mathrm{MA}_{\aleph_{1}}$ preserves Suslin's Hypothesis, and therefore Suslin's Hypothesis is consistent with $2^{\aleph_{0}}$ being singular of cofinality $\omega_{1}$.
} 
$\sigma$ to be the negation of Club Guessing. It follows from the above observation that the strategy of producing a model of $\sigma$ by building a finite support iteration in which the iterands have the c.c.c. cannot work. However, $\neg \mathrm{CG}$ is consistent. It follows from BPFA, and it can be forced over any model of $\mathrm{GCH}$ by a countable support iteration of proper forcings of length $\omega_{2}$. In fact, given a ladder system $\mathcal{A}=\left\langle A_{\delta}: \delta \in \operatorname{Lim}\left(\omega_{1}\right)\right\rangle$, the natural forcing for adding, by initial segments, a club $C \subseteq \omega_{1}$ 'avoiding' $\mathcal{A}$ - in the sense that $C \cap A_{\delta}$ is finite for all $\delta \in \operatorname{Lim}\left(\omega_{1}\right)$ - is proper 4 On the other hand, although countable support iterations of proper forcings are always proper, finite support iterations of infinite length of forcings that are not c.c.c. always collapse $\omega_{1}$. It follows that any (standard) forcing construction for producing a model of $\neg \mathrm{CG}$ by iterating instances of the proper forcing for adding clubs avoiding given ladder systems will have to be an iteration with countable supports rather than finite supports, and therefore will never give rise to a model with $2^{\aleph_{0}}>\aleph_{2}$. The reason is the well-known general fact that for any countable support iteration $\left\langle\mathbb{P}_{\xi}: \xi \leq \lambda\right\rangle$ of nontrivial forcings and any ordinal $\xi$, if $\lambda \geq \xi+\omega_{1}$, then $\mathbb{P}_{\lambda}$ forces over $V$ that there is a surjection from $\omega_{1}^{V}$ onto the reals of $V\left[\dot{G}_{\xi}\right]$ (where of course $\dot{G}_{\xi}$ denotes the canonical $\mathbb{P}_{\xi}$-name for the generic filter). In particular, if $c f(\lambda) \geq \omega_{1}$ and $\mathbb{P}_{\lambda}$ has the $\lambda$-c.c., then $\mathbb{P}_{\lambda}$ forces $2^{\aleph_{0}} \leq \aleph_{2}$. To sum up, c.c.c. forcing is useless when it comes to forcing the negation of club-guessing principles over models satisfying these club-guessing principles, and, on the other hand, countable support iterations of proper forcing notions can easily give rise to generic extensions satisfying the negation of club-guessing principles, but $2^{\aleph_{0}} \leq \aleph_{2}$ must necessarily hold in those extensions.

In view of these considerations it is natural to enquire whether various failures of Club Guessing on $\omega_{1}$ are consistent with the continuum large. In some cases, this question can be settled by taking a model of the property in question together with the continuum small and arguing that adding many Cohen or random reals to it preserves the property. For example, it can be proved that the very statement we have been considering above, namely $\neg \mathrm{CG}$, is indeed compatible with $2^{\aleph_{0}}>\aleph_{2}$. In fact it is not difficult to prove (and possibly folklore) that the product with finite supports of Cohen forcing always preserves $\neg \mathrm{CG}$.

There are other antidiamond principles for which the strategy of adding many Cohen reals does not work. This is for example the case for the negation of Weak Club Guessing. Weak Club Guessing (WCG) says that there exists a ladder system $\mathcal{A}=\left\langle A_{\delta}: \delta \in \operatorname{Lim}\left(\omega_{1}\right)\right\rangle$ with the property that for every club $C \subseteq \omega_{1}$ there is some $\delta \in C$ such that $A_{\delta} \cap C$ is infinite. Note that Club Guessing implies Weak Club Guessing and that, by what we have already mentioned, $\neg \mathrm{WCG}$ is a consequence of BPFA and can be forced over any model of GCH by a countable support iteration of proper forcings of length $\omega_{2}$. On the other hand, Cohen forcing always adds a ladder system witnessing WCG. This was originally proved by Juhasz in [9], where he showed that a weakening of \& implying Weak Club Guessing always holds after adding a Cohen real. Hence, the consistency of $\neg$ WCG with $2^{\aleph_{0}}>\aleph_{2}$ cannot be proved by adding Cohen reals to a model where Weak Club Guessing is false. However, one possibility for this is to add many random reals to a model of $\neg$ WCG. In fact, it is not hard to see (and, again, possibly folklore) that random forcing always preserves $\neg \mathrm{WCG}$.

\footnotetext{
${ }^{4}$ It follows from this that in fact the negation of the weaker principle known as Weak Club Guessing (see below) follows from BPFA and can be forced over any GCH model.
} 
There are however strengthenings of $\neg \mathrm{CG}$ for which the above methods do not work. Consider for example the conjunction of $\neg \mathrm{WCG}$ and $\mathrm{MA}_{\omega_{1}}$. This theory implies several strong forms of $\neg \mathrm{WCG} 5$ As we said, $\neg \mathrm{WCG}$ is preserved after adding random reals, but $\mathrm{MA}_{\omega_{1}}$ will fail in the resulting model 6

Another example of a strengthening of $\neg \mathrm{CG}$ that cannot be forced easily with a large continuum is the negation of $\mho$ (mho). The principle $\mho$, formulated by Moore, says that there is a sequence $\left\langle f_{\alpha}: \alpha \in \omega_{1}\right\rangle$ such that $f_{\alpha}$ is a continuous map, with respect to the order topology, from $\alpha$ into $\omega$ for all $\alpha \in \omega_{1}$, and with the property that for every club $E \subseteq \omega_{1}$ there is a $\delta$ in $E$ such that $f_{\delta}$ takes all values in $\omega$ on $E \cap \delta$. The following is an observation of Moore concerning this statement: Notice that if $\alpha<\omega_{1}$ and $f: \alpha \rightarrow \omega$ is continuous, then $\alpha$ can be partioned into clopen intervals on which $f$ is constant. In such a situation there is a cofinal $C \subseteq \alpha$ of order-type at most $\omega$ such that $f(\varepsilon)$ depends only on the size of $\varepsilon \cap C$. From this it is clear that $\mho$ follows from CG. In [16] Moore shows that $\mho$ implies the existence of an Aronszajn line containing no Countryman type, and asks whether $\mho$ follows from $2^{\aleph_{0}}>\aleph_{2}$. One motivation for this question is that, by the above implication, $\mho$ entails that there is no basis for the uncountable linear orders containing exactly 5 uncountable members 7 On the other hand, Moore proved in [15] that the existence of such a basis is consistent with ZFC and that in fact it follows from PFA that there is such a basis. Hence, if $\mho$ could be derived from $2^{\aleph_{0}}>\aleph_{2}$, then the existence of a 5 element basis for the uncountable linear orders would imply $2^{\aleph_{0}}=\aleph_{2}$ (it is easy to see that it implies $2^{\aleph_{0}}>\aleph_{1}$ ).

It should be noted that $\mathrm{MA}_{\omega_{1}}, \neg \mathrm{WCG}$ and $\neg \mho$ follow from the forcing axiom for the class of all proper posets of size $\aleph_{1}$ (which we will call $\operatorname{PFA}\left(\omega_{1}\right)$ ). In this paper we introduce an alternative method to standard countable support iterations for producing models of certain $\Pi_{2}$ statements. Using this method we prove that a certain forcing axiom which is a natural fragment of PFA $\left(\omega_{1}\right)$ and which implies the three statements above $-\mathrm{MA}_{\omega_{1}}, \neg \mathrm{WCG}$, and $\neg \mho-$ is consistent together with $2^{\aleph_{0}}>\aleph_{2} 8$ In fact, we build a cardinal-preserving generic extension where this fragment of $\operatorname{PFA}\left(\omega_{1}\right)$ holds and $2^{\aleph_{0}}$ is equal to $\kappa$, where $\kappa$ is an arbitrarily fixed cardinal satisfying certain GCH like assumptions in the ground model.

Definition 1.1. Given a poset $\mathbb{P}$, we will say that $\mathbb{P}$ is finitely proper if and only if for every regular cardinal $\lambda>|T C(\mathbb{P})|$, every finite set $\left\{N_{i}: i \in m\right\}$ of countable elementary substructures of $H(\lambda)$ containing $\mathbb{P}$ and every condition $p \in \bigcap\left\{N_{i}: i<m\right\} \cap \mathbb{P}$ there is a $\mathbb{P}$-condition extending $p$ and $\left(N_{i}, \mathbb{P}\right)$-generic for all $i$.

\footnotetext{
${ }^{5}$ One such strong form of $\neg \mathrm{WCG}$ is for instance Code(even-odd), a principle formulated by Miyamoto saying that for every ladder system $\mathcal{A}=\left\langle A_{\delta}: \delta \in \operatorname{Lim}\left(\omega_{1}\right)\right\rangle$ and every $B \subseteq \omega_{1}$ there are two clubs $C$ and $D$ of $\omega_{1}$ such that for each $\delta \in C$, if $\delta \in B$ (resp. $\delta \notin B$ ), then $\left|A_{\delta} \cap D\right|<\aleph_{0}$ is odd (resp. even).

${ }^{6}$ One reason why $\mathrm{MA}_{\omega_{1}}$ fails after adding random reals is that in the extension there is a c.c.c. partial order whose product with itself is not c.c.c. This result is due to Kunen and a proof can be found in [18].

${ }^{7}$ If there is such a basis for the uncountable linear orders, then there must be a Countryman type $C$ such that every Aronszajn line contains a copy of $C$ or of the reverse of $C$ (or of both).

${ }^{8}$ Concerning the connection previously mentioned between $\mho$ and the (non)existence of a 5 element basis for the uncountable linear orders, we should point out that it is still open whether the existence of such a basis is compatible with $2^{\aleph_{0}}>\aleph_{2}$.
} 
Definition 1.2. Let $\mathrm{PFA}^{\text {fin }}\left(\omega_{1}\right)$ denote the forcing axiom for the class $\Gamma$ of all finitely proper posets of size $\aleph_{1}$ and for families of $\aleph_{1}$-many dense sets. More precisely, $\mathrm{PFA}^{\mathrm{fin}}\left(\omega_{1}\right)$ says that whenever $\mathbb{P} \in \Gamma$ and $\left\{D_{\alpha}: \alpha \in \aleph_{1}\right\}$ is a set of dense subsets of $\mathbb{P}$, there is a filter $G$ on $\mathbb{P}$ meeting every $D_{\alpha}$.

Note that c.c.c. partial orders are finitely proper. Indeed, if $\mathbb{P}$ is c.c.c. and $N$ is any countable elementary substructure of $H(\lambda)$, for any cardinal $\lambda>|T C(\mathbb{P})|$, such that $\mathbb{P} \in N$, then any condition in $\mathbb{P}$ is $(N, \mathbb{P})$-generic, simply because $A \subseteq N$ for any maximal antichain of $\mathbb{P}$ in $N$. Therefore the forcing axiom $\operatorname{PFA}^{\mathrm{fin}}\left(\omega_{1}\right)$ is a generalization of $\mathrm{MA}_{\omega_{1}}$. Also, unlike any form of Martin's Axiom, $\mathrm{PFA}^{\text {fin }}\left(\omega_{1}\right)$ does have a strong impact on the club filter on $\omega_{1}$. Specifically, this forcing axiom implies the failure of both WCG and $\mho$. The proofs of these implications can be found in Section 5 .

Our main theorem is the following.

Theorem $1.3(\mathrm{CH})$. If $\kappa$ is a cardinal such that $\kappa^{\aleph_{1}}=\kappa$ and $2^{<\kappa}=\kappa$, then there exists a proper forcing notion $\mathcal{P}$ with the $\aleph_{2}$-chain condition such that both $\mathrm{PFA}^{f i n}\left(\omega_{1}\right)$ and $2^{\aleph_{0}}=\kappa$ hold in the generic extension by $\mathcal{P}$.

Our method produces a proper forcing notion with the $\aleph_{2}$-chain condition. This forcing notion $\mathcal{P}$ is the direct limit $\mathcal{P}_{\kappa}$ of a sequence $\left\langle\mathcal{P}_{\alpha}: \alpha<\kappa\right\rangle$ of partial orders, where $\mathcal{P}_{\alpha}$ is a complete suborder of $\mathcal{P}_{\beta}$ whenever $\alpha$ is less than $\beta$. Our construction can thus be seen as a forcing iteration in a broad sense.

One crucial feature in the proof of properness is the use of certain finite "symmetric systems" of countable structures as side conditions. These structures will be elementary substructures of $H(\kappa)$ and will be added by $\mathcal{P}_{0}$. If $N$ is one of them, $q=(F, \Delta) \in \mathcal{P}_{\alpha},(N, \alpha) \in \Delta$, and $N$ belongs to a club of "sufficiently correct" structures, then all relevant pieces of information coming from any $\mathcal{P}_{\alpha}$-extension of $q$ can be relativized to $N$. This is the case essentially because, under the above assumptions, if $\xi \in \operatorname{dom}(F)$ - i.e., $F(\xi)$ carries nontrivial information on the (finitely proper) poset $\Phi(\xi)$ with domain included in $\omega_{1}$ picked by our bookkeeping - and $\xi \in \alpha \cap N$, then $F(\xi)$ is asked to be generic over $N\left[\dot{G}_{\xi}\right]$ with respect to $\Phi(\xi)$. The domain of $F$ will be finite, and for every $\xi \in \operatorname{dom}(F), F(\xi)$ will be a $\mathcal{P}_{\xi}$-name for a countable ordinal. The general technique of ensuring properness of a given forcing notion by explicitly incorporating elementary substructures of some large enough model as side conditions may be traced back to Todorčević [23]. The more specific approach of considering symmetric systems of countable structures as side conditions in contexts in which one starts with a model of $\mathrm{CH}$ and wants to obtain a forcing notion which is proper and has the $\aleph_{2}-$ chain condition is quite natural. In fact, this approach has already shown up in several places in the literature prior to our work (see for example [1], [10] and [24]). The main novelty of our present work is that it incorporates the use of symmetric systems of structures as side conditions affecting all iterands of a given forcing iteration (or of an initial segment thereof) rather than a single forcing as in the above references. It is worth pointing out that Neeman ([17]) has developed a different method for building proper forcing notions by means of finite support iterations with side conditions. His side conditions are $\in$-chains of certain types of objects rather than symmetric systems of countable structures. The members of Neeman's side conditions may be countable elementary substructures but may also be of a different nature. One important 
difference between his work and ours is that in ours we strive to obtain a forcing notion with the $\aleph_{2}$-c.c., which is the reason why we cannot do with $\in$-chains of structures and need symmetric systems instead, whereas the $\aleph_{2}-$ c.c. typically does not hold in Neeman's constructions 9

We feel that the main contribution of the present paper is not so much a particular consistency result as the introduction of a fairly general method for building interesting forcing constructions. In fact, we have found further applications of (variations of) our method since this paper was first written and circulated in 2010. For instance, in [2] we build a model of a generalisation of Martin's Axiom to a certain natural class of forcing notions with the $\aleph_{2}$-chain condition, with no restriction on their size. This generalisation of Martin's Axiom implies certain interesting 'uniform' failures of Club Guessing whose consistency we don't know how to prove by methods other than ours.

There are natural weakenings of $\mho$ whose negation does not seem to hold in the models built by the methods in the present paper. Specifically, given $n<\omega$ let $\mho_{n}$ be the principle saying that there is a sequence $\left(f_{\delta}\right)_{\delta \in \omega_{1}}$ with $f_{\delta}: \delta \longrightarrow n$ continuous function for each $\delta$ such that for every club $C \subseteq \omega_{1}$ there is some $\delta \in C$ such that $f_{\delta}^{-1}(j) \cap C$ is unbounded in $\delta$ for all $j<n$. Clearly, for all $2 \leq n<m<\omega$, $\mho$ implies $\mho_{m}$, and $\mho_{m}$ implies $\mho_{n}$ (these weakenings of $\mho$ have also been defined by Moore). Our present methods do not seem to produce models of $\neg \mho_{n}$ for any $n$ (see Section 5 for a brief discussion of this). We should point out that, even if none of the principles $\neg \mho_{n}$ is known to follow from $\mathrm{PFA}^{\mathrm{fin}}\left(\omega_{1}\right)$, already $\neg \mho_{2}$ certainly follows from $\mathrm{PFA}\left(\omega_{1}\right)$ (see the remark in Section [5).

The rest of the paper is organized as follows: In Section 2 we introduce the notion of symmetric system of structures and prove basic properties of this notion that we will use throughout the paper. In Section 3 we present a rather general construction of a finite support forcing iteration using symmetric systems of structures as side conditions and prove several facts applying to this general context. Section 4 starts with the definition of a partial order $\mathcal{P}$ that will be shown to witness the conclusion of Theorem 1.3. This partial order is a special case of the construction in Section 3 . We then prove the relevant facts of $\mathcal{P}$ not covered by the general theory in Section 3. It follows from these facts that $\mathcal{P}$ indeed witnesses the conclusion of Theorem 1.3. Finally, in Section 5] we show that $\mathrm{PFA}^{\text {fin }}\left(\omega_{1}\right)$ implies the failure of Weak Club Guessing and of $\mho$.

Even if this work tries to be reasonably self-contained, we will assume that the reader has a good knowledge of forcing, and in particular some familiarity with proper forcing. Two good references are Kunen ([11]) and Jech ([8]). Most of our notation is standard, and we have tried to give complete explanations of the relevant symbols and notions whenever we deviate from the standard use.

\section{Symmetric SYSTEMS}

Our forcing $\mathcal{P}$ for proving Theorem 1.3, to be defined in Section 4, will be the direct limit $\mathcal{P}_{\kappa}$ of a certain sequence $\left\langle\mathcal{P}_{\alpha}: \alpha<\kappa\right\rangle$ of forcings. The properness of each $\mathcal{P}_{\alpha}$ will be witnessed by a certain club $\mathcal{M}_{\alpha}^{*}$ of $\left[H\left(\theta_{\alpha}\right)\right]^{\aleph_{0}}$ for some high enough cardinal $\theta_{\alpha}$ (see Section 44). The main idea here is to use the elements of $\mathcal{M}_{\alpha}$ - where $\mathcal{M}_{\alpha}$ is the club of restrictions to $H(\kappa)$ of members of $\mathcal{M}_{\alpha}^{*}$ - as side

\footnotetext{
${ }^{9}$ He typically does need some $\kappa$-c.c., for larger $\kappa$, which he tends to achieve thanks to the use of structures of the form $H(\alpha)$ in his side conditions.
} 
conditions to ensure properness, but without losing the $\aleph_{2}$-chain condition. This brings us to the notion of a symmetric system of structures. As we mentioned in the introduction, the notion of a symmetric system of structures is a natural one in the context of building forcing notions, over models of $\mathrm{CH}$, which are intended to be both proper and with the $\aleph_{2}$-chain condition. In this section we define this notion and analyse its basic properties, which we will repeatedly use throughout the rest of the paper. This type of analysis can be found also for example in [1, [10] and 24, where the notion of a symmetric system shows up too (with different names).

Here, and in the remainder of the paper, we adopt the convention of denoting by $\delta_{N}$ the ordinal $N \cap \omega_{1}$ if $N$ is a set such that $N \cap \omega_{1}$ is an ordinal. Also, in this section $\kappa$ can be taken to be the same $\kappa$ that has been fixed in the statement of Theorem 1.3. but everything here works the same with any other choice of $\kappa$ (as long as $\kappa \geq \omega_{2}$ and $\kappa$ is a cardinal) 10

Definition 2.1. Let $P \subseteq H(\kappa)$, and let $\left\{N_{i}: i<m\right\}$ be a finite set of countable subsets of $H(\kappa)$. We will say that $\left\{N_{i}: i<m\right\}$ is a P-symmetric system if

(A) For every $i<m,\left(N_{i}, \in, P\right)$ is an elementary substructure of $(H(\kappa), \in, P)$.

(B) Given distinct $i, i^{\prime}$ in $m$, if $\delta_{N_{i}}=\delta_{N_{i^{\prime}}}$, then there is a (unique) isomorphism

$$
\Psi_{N_{i}, N_{i^{\prime}}}:\left(N_{i}, \in, P\right) \longrightarrow\left(N_{i^{\prime}}, \in, P\right) .
$$

Furthermore, we ask that $\Psi_{N_{i}, N_{i^{\prime}}}$ be the identity on $N_{i} \cap N_{i^{\prime}}$.

(C) For all $i, j$ in $m$, if $\delta_{N_{j}}<\delta_{N_{i}}$, then there is some $i^{\prime}<m$ such that $\delta_{N_{i^{\prime}}}=\delta_{N_{i}}$ and $N_{j} \in N_{i^{\prime}}$.

(D) For all $i, i^{\prime}, j$ in $m$, if $N_{j} \in N_{i}$ and $\delta_{N_{i}}=\delta_{N_{i^{\prime}}}$, then there is some $j^{\prime}<m$ such that $\Psi_{N_{i}, N_{i^{\prime}}}\left(N_{j}\right)=N_{j^{\prime}}$.

In $(A)$ in the above definition, and elsewhere, we will tend to refer to structures $(N, \in, P \cap N)$ by the simpler expression $(N, \in, P)$.

Lemma 2.2. Let $P \subseteq H(\kappa)$ and let $N, N^{\prime}$ and $M$ be countable elementary substructures of $(H(\kappa), \in, P)$. Suppose $M \in N$ and $\Psi_{N, N^{\prime}}:(N, \in, P) \longrightarrow\left(N^{\prime}, \in, P\right)$ is an isomorphism. Then $\Psi_{N, N^{\prime}}(M)$ is also a countable elementary substructure of $(H(\kappa), \in, P)$.

Proof. First note that $\Psi_{N, N^{\prime}} \uparrow M$ is an isomorphism between $(M, \in, P)$ and $\left(\Psi_{N, N^{\prime}}(M), \in, P\right)$. Assume now that $\vec{y}$ is a finite vector of elements of $\Psi_{N, N^{\prime}}(M)$ and that the formula $\exists x \varphi(x, \vec{y})$ is true in $(H(\kappa), \in, P)$. We show that there is some $z \in \Psi_{N, N^{\prime}}(M)$ such that $(H(\kappa), \in, P)=\varphi(z, \vec{y})$. But $\vec{y}$ is also a finite vector of elements of $N^{\prime}$, and by correctness of $\left(N^{\prime}, \in, P\right)$, the formula $\exists x \varphi(x, \vec{y})$ is true in $\left(N^{\prime}, \in, P\right)$. Let $\overleftarrow{y}$ be the vector of elements of $N$ such that $\Psi_{N, N^{\prime}}(\overleftarrow{y})=\vec{y}$, and note that the formula $\exists x \varphi(x, \overleftarrow{y})$ is true in $(N, \in, P)$ since the map $\Psi_{N, N^{\prime}}^{-1}:\left(N^{\prime}, \in\right.$ $P) \longrightarrow(N, \in, P)$ is an isomorphism. Furthermore, by correctness of $(N, \in, P)$, the formula $\exists x \varphi(x, \overleftarrow{y})$ is also true in $(H(\kappa), \in, P)$. From this, and using the fact that $\overleftarrow{y}$ is also a vector of elements of $M$, we conclude that there exists an element $z^{\prime}$ in $M$ such that the formula $\varphi\left(z^{\prime}, \overleftarrow{y}\right)$ is true in $(M, \in, P)$. It suffices to let $z=\Psi_{N, N^{\prime}}\left(z^{\prime}\right)$.

Lemma 2.3. Let $P \subseteq H(\kappa)$, let $\mathcal{N}$ be a P-symmetric system, and let $N \in \mathcal{N}$.

\footnotetext{
${ }^{10}$ The theory works also for the case $\kappa=\omega_{1}$, but this is a degenerate case in which symmetric systems are simply finite $\in$-chains of countable transitive models.
} 
(i) If $M_{1} \in \mathcal{N} \cap N$ and there is some $M_{2} \in \mathcal{N}$ (not necessarily in $\mathcal{N} \cap N$ ) such that $\delta_{M_{1}}<\delta_{M_{2}}<\delta_{N}$, then there is some $M_{3}$ in $\mathcal{N} \cap N$ such that $M_{1} \in M_{3}$ and $\delta_{M_{3}}=\delta_{M_{2}}$.

(ii) In particular, $\mathcal{N} \cap N$ is also a $P$-symmetric system.

(iii) If $\mathcal{W} \subseteq N$ is a $P$-symmetric system and $\mathcal{N} \cap N \subseteq \mathcal{W}$, then

$$
\mathcal{V}:=\mathcal{N} \cup\left\{\Psi_{N, N^{\prime}}(W): W \in \mathcal{W}, N^{\prime} \in \mathcal{N}, \delta_{N^{\prime}}=\delta_{N}\right\}
$$

is a $P$-symmetric system.

Proof. We start with (i). Since $\mathcal{N}$ satisfies clause $(C)$ in the definition of a symmetric system, there exist some $M_{4}$ and $N^{\prime}$ in $\mathcal{N}$ such that $M_{1} \in M_{4} \in N^{\prime}, \delta_{M_{4}}=\delta_{M_{2}}$, and $\delta_{N^{\prime}}=\delta_{N}$. Since $\left(N^{\prime}, \in, P\right)$ and $(N, \in, P)$ are isomorphic and the corresponding isomorphism $\Psi_{N^{\prime}, N}$ fixes $N \cap N^{\prime}$ (in particular, it fixes $M_{1}$ ), it follows that $M_{1} \in \Psi_{N^{\prime}, N}\left(M_{4}\right)$. Finally, note that clause $(D)$ implies that $M_{3}:=\Psi_{N^{\prime}, N}\left(M_{4}\right)$ is in $\mathcal{N} \cap N$, and that $\delta_{M_{3}}=\delta_{M_{4}}=\delta_{M_{2}}$ again as $\Psi_{N^{\prime}, N}$ fixes $N \cap N^{\prime}$.

Let us move on to conclusion (ii). $\mathcal{N} \cap N$ satisfies clauses $(A),(B)$ and $(D)$ of Definition 2.1 since they hold for $\mathcal{N}$, and $(C)$ follows from (i).

We prove now (iii). By Lemma 2.2. $\mathcal{V}$ satisfies clause $(A)$ of Definition 2.1.

Let us now check that $\mathcal{V}$ satisfies $(B)$. So, let $V_{1}$ and $V_{2}$ in $\mathcal{V}$ be such that $\delta_{V_{1}}=\delta_{V_{2}}$. We must show that $\left(V_{1}, \in, P\right)$ and $\left(V_{2}, \in, P\right)$ are isomorphic and that the corresponding isomorphism fixes $V_{1} \cap V_{2}$. Without loss of generality we can assume that $\delta_{V_{1}}<\delta_{N}$ (note that if $\delta_{V_{1}} \geq \delta_{N}$, then $V_{1}$ and $V_{2}$ are elements of $\mathcal{N})$ and that $V_{1}$ or $V_{2}$ are not in $N$. Note also that $\mathcal{V} \cap N \subseteq \mathcal{W}$ : If $W \in \mathcal{W}$, $N^{\prime} \in \mathcal{N}, \delta_{N^{\prime}}=\delta_{N}$, and $\Psi_{N, N^{\prime}}(W) \in N$, then $\Psi_{N, N^{\prime}}(W) \in N \cap N^{\prime}$, and therefore $\Psi_{N, N^{\prime}}(W)=W$ as $\Psi_{N, N^{\prime}}$ is the identity on $N \cap N^{\prime}$. It follows that there are $N_{1}$ and $N_{2}$, both in $\mathcal{N}$, such that $\delta_{N_{i}}=\delta_{N}$ and $V_{i} \in N_{i}(i \in\{1,2\})$. Let $V_{i}^{\prime}=$ $\Psi_{N_{i}, N}\left(V_{i}\right)(i \in\{1,2\})$. It follows that the map $\Psi_{N, N_{2}} \circ \Psi_{V_{1}^{\prime}, V_{2}^{\prime}} \circ\left(\Psi_{N_{1}, N} \uparrow V_{1}\right)$ is an isomorphism $\Psi_{V_{1}, V_{2}}$ between $\left(V_{1}, \in, P\right)$ and $\left(V_{2}, \in, P\right)$, and of course it is the unique isomorphism between these structures. Let $\tilde{V}_{1}=\Psi_{N_{1}, N_{2}}\left(V_{1}\right)=\Psi_{N, N_{2}}\left(V_{1}^{\prime}\right)$, and note that $\Psi_{V_{1}, V_{2}}=\Psi_{\tilde{V}_{1}, V_{2}} \circ\left(\Psi_{N_{1}, N_{2}} \uparrow V_{1}\right)$. To see that $\Psi_{V_{1}, V_{2}}$ fixes $V_{1} \cap V_{2}$, note that if $x \in V_{1} \cap V_{2}$, then $x=\Psi_{N_{1}, N_{2}}(x)=x$ (since $x \in N_{1} \cap N_{2}$ ), and therefore $\Psi_{V_{1}, V_{2}}(x)=\Psi_{\tilde{V}_{1}, V_{2}}(x)=x$. The last equality holds since $x \in \tilde{V}_{1} \cap V_{2}$ and since $\Psi_{\tilde{V}_{1}, V_{2}}$ fixes $\tilde{V}_{1} \cap V_{2}$ (this is true since $\Psi_{\tilde{V}_{1}, V_{2}}=\Psi_{N, N_{2}}\left(\Psi_{V_{1}^{\prime}, V_{2}^{\prime}}\right)$ and since $\Psi_{V_{1}^{\prime}, V_{2}^{\prime}}$ fixes $\left.V_{1}^{\prime} \cap V_{2}^{\prime}\right)$.

The next step is to prove that $\mathcal{V}$ satisfies $(C)$. So, assume that $V_{1}, V_{2}$ are elements of $\mathcal{V}$ such that $\delta_{V_{1}}<\delta_{V_{2}}$. We must show that there is a $V_{3}$ in $\mathcal{V}$ containing $V_{1}$ and such that $\delta_{V_{2}}=\delta_{V_{3}}$. Note that if $\delta_{V_{1}} \geq \delta_{N}$, then $V_{1}$ and $V_{2}$ are elements of the symmetric system $\mathcal{N}$ and we are done. Also note that if $\delta_{V_{2}} \geq \delta_{N}>\delta_{V_{1}}$, then $V_{2} \in \mathcal{N}$ and there is some $N_{3}$ in $\mathcal{N}$ such that $V_{1} \in N_{3}$ and $\delta_{N_{3}}=\delta_{N}$. In particular there is some $V_{3} \in \mathcal{N}$ such that $\delta_{V_{3}}=\delta_{V_{2}}$ and $N_{3} \in V_{3}$, and therefore such that $V_{1} \in V_{3}$. So, we may assume that $\delta_{V_{2}}<\delta_{N}$ and that there are $W_{1}, W_{2}$ in $\mathcal{W}$, together with $N_{1}, N_{2}$ in $\mathcal{N}$, such that $V_{1}=\Psi_{N, N_{1}}\left(W_{1}\right)$ and $V_{2}=\Psi_{N, N_{2}}\left(W_{2}\right)$. Since $\mathcal{W}$ is a symmetric system, there exists some $W_{3} \in \mathcal{W}$ such that $W_{1} \in W_{3}$ and $\delta_{W_{3}}=\delta_{W_{2}}$. It suffices to let $V_{3}=\Psi_{N, N_{1}}\left(W_{3}\right)$.

Finally, we check that $\mathcal{V}$ satisfies $(D)$. Let $V_{1}, V_{2}$ and $V_{3}$ be elements of $\mathcal{V}$ such that $\delta_{V_{1}}<\delta_{V_{2}}=\delta_{V_{3}}$ and $V_{1} \in V_{2}$. We must show that $\Psi_{V_{2}, V_{3}}\left(V_{1}\right) \in \mathcal{V}$. Note that if $\delta_{V_{1}} \geq \delta_{N}$, then $V_{1}, V_{2}$ and $V_{3}$ are in $\mathcal{N}$ and we are done. So, we can assume that $\delta_{V_{1}}<\delta_{N}$. Now note that if $\delta_{V_{1}}<\delta_{N} \leq \delta_{V_{2}}$, then there are $N_{2}, N_{3} \in \mathcal{N}$ and $W \in \mathcal{W}$ such that $V_{1}=\Psi_{N, N_{2}}(W)$ and such that, for all $j \in\{2,3\}, \delta_{N_{j}}=\delta_{N}$ and 
$N_{j} \subseteq V_{j}$, and $N_{3}=\Psi_{V_{2}, V_{3}}\left(N_{2}\right)$ if $\delta_{N_{2}}<\delta_{V_{2}}$. Hence, $\Psi_{V_{2}, V_{3}}\left(V_{1}\right)=\Psi_{N_{2}, N_{3}}\left(V_{1}\right)=$ $\Psi_{N_{2}, N_{3}}\left(\Psi_{N, N_{2}}(W)\right)=\Psi_{N, N_{3}}(W) \in \mathcal{V}$. The last case of this proof is when $\delta_{V_{2}}<\delta_{N}$. In this case there are $W_{i} \in \mathcal{W}$ and $N_{i} \in \mathcal{N}(i \in\{1,2,3\})$ such that $\delta_{N_{i}}=\delta_{N}$ and $V_{i}=\Psi_{N, N_{i}}\left(W_{i}\right)$. Furthermore, since $V_{1} \in N_{1} \cap N_{2}$ and $\Psi_{N_{1}, N_{2}}$ fixes $N_{1} \cap N_{2}$, we also have that $\Psi_{N_{2}, N}\left(V_{1}\right)=\Psi_{N_{2}, N}\left(\Psi_{N_{1}, N_{2}}\left(V_{1}\right)\right)=\Psi_{N_{1}, N}\left(V_{1}\right)=W_{1}$. Since $\mathcal{W}$ is a symmetric system, we know that $W_{4}:=\Psi_{W_{2}, W_{3}}\left(W_{1}\right)$ is in $\mathcal{W}$. It thus follows that

$$
\begin{aligned}
\Psi_{V_{2}, V_{3}}\left(V_{1}\right) & =\Psi_{W_{3}, V_{3}}\left(\Psi_{W_{2}, W_{3}}\left(\left(\Psi_{V_{2}, W_{2}}\left(V_{1}\right)\right)\right)\right. \\
& =\Psi_{W_{3}, V_{3}}\left(\Psi_{W_{2}, W_{3}}\left(\Psi_{N_{2}, N}\left(V_{1}\right)\right)\right)=\Psi_{W_{3}, V_{3}}\left(\Psi_{W_{2}, W_{3}}\left(W_{1}\right)\right) \\
& =\Psi_{N, N_{3}}\left(W_{4}\right) \in \mathcal{V} .
\end{aligned}
$$

(The second equality is true since $\Psi_{V_{2}, W_{2}}$ is the same thing as $\Psi_{N_{2}, N} \uparrow V_{2}$, the third equality is true since $\Psi_{N_{2}, N}\left(V_{1}\right)=W_{1}$, and the last equality is true since $W_{4}=\Psi_{W_{2}, W_{3}}\left(W_{1}\right)$ and since $\Psi_{W_{3}, V_{3}}$ is the restriction of $\Psi_{N, N_{3}}$ to $W_{3}$.)

Lemma 2.4. Let $P \subseteq H(\kappa)$ and let $\mathcal{N}_{0}=\left\{N_{i}^{0}: i<m\right\}$ and $\mathcal{N}_{1}=\left\{N_{i}^{1}: i<m\right\}$ be $P$-symmetric systems. Suppose that $\left(\cup \mathcal{N}_{0}\right) \cap\left(\bigcup \mathcal{N}_{1}\right)=X$ and that there is an isomorphism $\Psi$ between the structures $\left\langle\bigcup_{i<m} N_{i}^{0}, \in, P, X, N_{i}^{0}\right\rangle_{i<m}$ and $\left\langle\bigcup_{i<m} N_{i}^{1}, \in\right.$, $\left.P, X, N_{i}^{1}\right\rangle_{i<m}$ fixing $X$. Then $\mathcal{N}_{0} \cup \mathcal{N}_{1}$ is a $P$-symmetric system.

Proof. Obviously, $\mathcal{N}_{0} \cup \mathcal{N}_{1}$ is a finite set of countable elementary substructures of $(H(\kappa), \in, P)$. We will check that this union also satisfies clauses $(B),(C)$ and $(D)$ of Definition 2.1.

We start with clause $(B)$. We must show that if $i_{0}, i_{1}<m$ are such that $\delta_{N_{i_{0}}^{0}}=\delta_{N_{i_{1}}^{1}}$, then the isomorphism $\Psi_{N_{i_{0}}^{0}, N_{i_{1}}^{1}}:=\Psi \circ \Psi_{N_{i_{0}}^{0}, N_{i_{1}}^{0}}$ fixes $N_{i_{0}}^{0} \cap N_{i_{1}}^{1}$. Now, if $x \in N_{i_{0}}^{0} \cap N_{i_{1}}^{1}$, then $x \in X \cap N_{i_{0}}^{0}$, which implies that $\Psi(x)=x \in N_{i_{0}}^{1} \cap N_{i_{1}}^{1}$ as $\Psi$ is an isomorphism between the structures $\left\langle\bigcup_{i<m} N_{i}^{0}, \in, X, N_{i}^{0}\right\rangle_{i<m}$ and $\left\langle\bigcup_{i<m} N_{i}^{1}, \in\right.$, $\left.X, N_{i}^{1}\right\rangle_{i<m}$. But then $x \in N_{i_{0}}^{0} \cap N_{i_{1}}^{0}$ again by the fact that $\Psi$ is an isomorphism between $\left\langle\bigcup_{i<m} N_{i}^{0}, \in, X, N_{i}^{0}\right\rangle_{i<m}$ and $\left\langle\bigcup_{i<m} N_{i}^{1}, \in, X, N_{i}^{1}\right\rangle_{i<m}$, which implies that $\Psi_{N_{i_{0}}^{0}, N_{i_{1}}^{0}}(x)=x$ and hence that $\left(\left(\Psi \uparrow N_{i_{1}}^{0}\right) \circ \Psi_{N_{i_{0}}^{0}, N_{i_{1}}^{0}}\right)(x)=\Psi_{N_{i_{0}}^{0}, N_{i_{1}}^{1}}(x)=x$.

As for $(C)$, it suffices to note that the existence of $\Psi$ implies that $\left\{\delta_{N_{i}^{0}}: i<\right.$ $m\}=\left\{\delta_{N_{i}^{1}}: i<m\right\}$.

Finally, we check that $\mathcal{N}_{0} \cup \mathcal{N}_{1}$ satisfies $(D)$ of Definition 2.1. So, let $M_{1}, M_{2}$, $M_{3} \in \mathcal{N}_{0} \cup \mathcal{N}_{1}$ be such that $M_{2} \in M_{1}$ and $\delta_{M_{1}}=\delta_{M_{3}}$. We must verify that $\Psi_{M_{1}, M_{3}}\left(M_{2}\right)$ is also in $\mathcal{N}_{0} \cup \mathcal{N}_{1}$. Without loss of generality we may assume that there are indices $i, j \in\{1,2,3\}$ such that $M_{i} \in \mathcal{N}_{0}$ and $M_{j} \in \mathcal{N}_{1}$ (otherwise, the proof follows from the fact that $\mathcal{N}_{0}$ and $\mathcal{N}_{1}$ satisfy clause $(D)$ ). The case when $M_{1}$ and $M_{3}$ are both in $\mathcal{N}_{1}$ and $M_{2}$ is in $\mathcal{N}_{0}$ can be treated as follows. First note that there exist some $i_{1}$ and $i_{2}$ such that $M_{1}=N_{i_{1}}^{1}$ and $M_{2}=N_{i_{2}}^{0}$. As $M_{2} \in M_{1}$ and $\Psi$ is an isomorphism fixing $X$ (in particular, $M_{2}$ ), $N_{i_{2}}^{0} \in N_{i_{1}}^{0}$. But $\Psi_{N_{i_{2}}^{0}, N_{i_{2}}^{1}}=\Psi_{N_{i_{1}}^{0}, N_{i_{1}}^{1}} \uparrow N_{i_{2}}^{0}$, and this isomorphism also fixes $M_{2}$. So, $M_{1}, M_{2}=N_{i_{2}}^{1}$ and $M_{3}$ are elements of $\mathcal{N}_{1}$. The last case that needs to be considered is when $M_{3}$ is in $\mathcal{N}_{0}$ and $M_{1}$ is in $\mathcal{N}_{1}$. Just as before, we can ensure the existence of $i_{1}$, $i_{2}$ and $i_{3}$ such that $M_{3}=N_{i_{3}}^{0}, M_{1}=N_{i_{1}}^{1}$ and $M_{2}=N_{i_{2}}^{1}$. Let $i_{4}$ be such that $N_{i_{4}}^{1}=\Psi_{N_{i_{1}}^{1}, N_{i_{3}}^{1}}\left(N_{i_{2}}^{1}\right)$ (recall that $\mathcal{N}_{1}$ satisfies clause $(D)$ of Definition 2.1) and note that $N_{i_{4}}^{0}=\Psi_{M_{1}, M_{3}}\left(M_{2}\right)$. 


\section{Finite SUPPORT FORCING ITERATIONS WITH SYMMETRIC SYSTEMS AS SIDE CONDITIONS}

In this section we describe a general construction of a $\kappa$-sequence $\left\langle\mathcal{P}_{\alpha}: \alpha \leq \kappa\right\rangle$ of partial orders. This construction will depend on a predicate $P \subseteq H(\kappa)$, to be fixed at the outset, together with both a sequence $\left\langle\dot{\mathcal{Q}}_{\alpha}: \alpha<\kappa\right\rangle$ such that each $\dot{\mathcal{Q}}_{\alpha}$ is a $\mathcal{P}_{\alpha}$-name for a poset on $\kappa$ and a sequence $\left\langle\dot{\mathcal{R}}_{\alpha}: \alpha<\kappa\right\rangle$ such that each $\dot{\mathcal{R}}_{\alpha}$ is a $\mathcal{P}_{\alpha}$-name for a relation included in $\left([H(\kappa)]^{\aleph_{0}}\right)^{V} \times \dot{\mathcal{Q}}_{\alpha}$ satisfying a certain closure property 11 As in Section 2, $\kappa$ can be taken in this section to be any cardinal at least $\omega_{2}$.

Let $P \subseteq H(\kappa)$ be a fixed predicate. We are going to describe what it means for a sequence $\left\langle\left(\mathcal{P}_{\alpha}, \leq_{\alpha}\right): \alpha \leq \kappa\right\rangle$ of partial orders to be the finite support iteration with $P$-symmetric systems as side conditions based on $\left\langle\left(\dot{\mathcal{Q}}_{\alpha}, \dot{\mathcal{R}}_{\alpha}\right): \alpha<\kappa\right\rangle$. This description will stretch up until Subsection 3.1. As we will see, the description will specify one unique object (for fixed parameters $P$ and $\left\langle\left(\dot{\mathcal{Q}}_{\alpha}, \dot{\mathcal{R}}_{\alpha}\right): \alpha<\kappa\right\rangle$ ). Hence our use above of the article 'the'.

To start with, $\mathcal{P}_{0}$ must consist of all pairs of the form

(a) $\left(\emptyset,\left\{\left(N_{i}, 0\right): i<m\right\}\right)$, where $\left\{N_{i}: i<m\right\}$ is a $P$-symmetric system.

Given $\mathcal{P}_{0}$-conditions $q_{\epsilon}=\left(\emptyset,\left\{\left(N_{i}^{\epsilon}, 0\right): i<m_{\epsilon}\right\}\right)$ for $\epsilon \in\{0,1\}, q_{1} \leq_{0} q_{0}$ if $\left\{N_{i}^{0}: i<m_{0}\right\} \subseteq\left\{N_{i}^{1}: i<m_{1}\right\}$.

In the definition of a $\mathcal{P}_{0}$-condition we have used the empty set in a completely vacuous way. These (vacuous) $\emptyset$ 's are there to ensure that the (uniformly defined) operation of restricting a condition in a (further) $\mathcal{P}_{\alpha}$ to an ordinal $\beta<\alpha$ yields a condition in $\mathcal{P}_{\beta}$ when applied to any condition in any $\mathcal{P}_{\alpha}$ and to $\beta=0$.

Notation 3.1. If $q$ is an ordered pair, we denote the first component of $q$ by $F_{q}$ and the second component of $q$ by $\Delta_{q}$. Also, if $q$ is an ordered pair such that $F_{q}$ is a function and $\Delta_{q}$ is a relation and $\xi$ is an ordinal, the restriction of $q$ to $\xi$, denoted by $\left.q\right|_{\xi}$, is defined as the pair

$$
\left.q\right|_{\xi}:=\left(F_{q} \uparrow \xi,\left\{(N, \min \{\beta, \xi\}):(N, \beta) \in \Delta_{q}\right\}\right) .
$$

Let $\alpha \leq \kappa, \alpha>0$, and suppose that we have defined $\mathcal{P}_{\xi}$ for all $\xi<\alpha$. Suppose also that if $\xi<\alpha$ and $q \in \mathcal{P}_{\xi}$, then $q$ is an ordered pair of the form $\left(F_{q}, \Delta_{q}\right)$, where

- $F_{q}$ is a finite function with domain included in $\xi$, and

- $\Delta_{q}$ is a finite relation $\left\{\left(N_{i}, \tau_{i}\right): i \in n\right\}$ such that $\operatorname{dom}\left(\Delta_{q}\right)=\left\{N_{i}: i<n\right\}$ is a $P$-symmetric system and, for all $i, \tau_{i}$ is an ordinal such that $\tau_{i} \leq \xi$.

If $\alpha=\sigma+1$, we require that the following hold:

(1) $\dot{\mathcal{Q}}_{\sigma}$ is a $\mathcal{P}_{\sigma}$-name for a partial order.

(2) $\dot{\mathcal{R}}_{\sigma}$ is a $\mathcal{P}_{\sigma}$-name for a relation $R$ such that

$$
R \subseteq\left([H(\kappa)]^{\aleph_{0}}\right)^{V} \times \dot{\mathcal{Q}}_{\sigma}
$$

and such that $\left(N, x^{\prime}\right) \in R$ whenever $(N, x) \in R$ and $x^{\prime}$ is a $\dot{\mathcal{Q}}_{\sigma}$-condition extending $x$ ( $R$ is downward closed with respect to $\left.\dot{\mathcal{Q}}_{\sigma}\right)$.

The definition of $\mathcal{P}_{\alpha}$ is as follows (regardless of whether $\alpha$ is a successor or a limit ordinal). Conditions in $\mathcal{P}_{\alpha}$ are pairs of the form

$$
q=\left(F_{q}, \Delta_{q}\right)
$$

\footnotetext{
${ }^{11}$ In our application, in Section $4 \dot{\mathcal{R}}_{\alpha}$ will be the name for a relation holding about a typical pair $(N, \nu)$ exactly when $\nu$ is an $\left(N\left[\dot{G}_{\alpha}\right], \dot{\mathcal{Q}}_{\alpha}\right)$-generic condition.
} 
with the following properties:

(b0) $F_{q}$ is a finite function with $\operatorname{dom}\left(F_{q}\right) \subseteq \alpha$.

(b1) $\Delta_{q}$ is of the form $\left\{\left(N_{i}, \beta_{i}\right): i<m\right\}$ where, for all $i<m$, $\beta_{i} \leq \alpha \cap \sup \left(N_{i} \cap \kappa\right) 12$

(b2) For all $\xi<\alpha$, the restriction of $q$ to $\xi$ is a condition in $\mathcal{P}_{\xi}$.

(b3) If $\xi \in \operatorname{dom}\left(F_{q}\right)$, then $F_{q}(\xi)$ is a $\mathcal{P}_{\xi}$-name and $\left.q\right|_{\xi} \Vdash_{\xi} F_{q}(\xi) \in \dot{\mathcal{Q}}_{\xi}$.

(b4) If $\xi \in \operatorname{dom}\left(F_{q}\right),(N, \beta) \in \Delta_{q}, \beta \geq \xi+1$, and $\xi \in N$, then

$$
\left.q\right|_{\xi} \Vdash_{\xi}\left(N, F_{q}(\xi)\right) \in \dot{\mathcal{R}}_{\xi}
$$

Given conditions

$$
q^{\epsilon}=\left(F_{\epsilon},\left\{\left(N_{i}^{\epsilon}, \beta_{i}^{\epsilon}\right): i<m_{\epsilon}\right\}\right)
$$

(for $\epsilon \in\{0,1\}$ ) in $\mathcal{P}_{\alpha}$, we will say that $q^{1} \leq_{\alpha} q^{0}$ if and only if the following holds:

(c1) For all $\xi<\alpha,\left.q^{1}\right|_{\xi} \leq\left._{\xi} q^{0}\right|_{\xi}$.

(c2) $\operatorname{dom}\left(F_{0}\right) \subseteq \operatorname{dom}\left(F_{1}\right)$ and, for all $\xi \in \operatorname{dom}\left(F_{0}\right)$,

$$
\left.q^{1}\right|_{\xi} \Vdash_{\xi} F_{1}(\xi) \leq_{\dot{\mathcal{Q}}_{\xi}} F_{0}(\xi) .
$$

(c3) For all $i<m_{0}$ there is some $\widetilde{\beta}_{i} \geq \beta_{i}^{0}$ such that $\left(N_{i}^{0}, \widetilde{\beta}_{i}\right) \in \Delta_{q^{1}}$.

Notation 3.2. Given $\alpha \leq \kappa$ and a $\mathcal{P}_{\alpha}$-condition $q=\left(F_{q}, \Delta_{q}\right), \operatorname{dom}\left(F_{q}\right)$ will also be denoted by $\operatorname{supp}(q)$ and will be called the support of $q$.

Note that if $\alpha<\beta \leq \kappa$, then $\mathcal{P}_{\alpha} \subseteq \mathcal{P}_{\beta}$ and every $\mathcal{P}_{\beta}$-condition $q=\left(F,\left\{\left(N_{j}, \beta_{j}\right)\right.\right.$ : $j<m\}$ ) such that $\operatorname{supp}(q) \subseteq \alpha$ and $\beta_{j} \leq \alpha$ for all $j$ is also a $\mathcal{P}_{\alpha}$-condition and is in fact equal to its restriction to $\alpha$.

Also note that if $\alpha$ is a nonzero limit ordinal, then a pair $q=\left(F_{q}, \Delta_{q}\right)$ is a $\mathcal{P}_{\alpha}-$ condition if and only if it satisfies $(b 0)-(b 2)$.

We will sometimes talk about 'finite support iterations with $P$-symmetric systems as side conditions' in contexts where the sequence $\left\langle\left(\dot{\mathcal{Q}}_{\alpha}, \dot{\mathcal{R}}_{\alpha}\right): \alpha<\kappa\right\rangle$ is irrelevant. We may also omit $P$ when it is not relevant.

3.1. General facts. In this subsection we present several facts that all finite support iterations with $P$-symmetric systems as side conditions will satisfy.

In arguments involving this type of construction (for example in the proof of Theorem 1.3) one naturally finds oneself having to prove that natural amalgamations of conditions are themselves conditions. The following five lemmas give some basic properties of such amalgamations that are often used in those types of arguments.

We start with our first amalgamation lemma. An immediate consequence of this lemma (Corollary 3.4) is that our use of the term "iteration" is appropriate; more specifically, it follows from the lemma that if $\left\langle\mathcal{P}_{\xi}: \xi \leq \kappa\right\rangle$ is a finite support iteration with $P$-symmetric systems as side conditions, then it is a forcing iteration in the sense that $\mathcal{P}_{\alpha}$ is a complete suborder of $\mathcal{P}_{\beta}$ whenever $\alpha<\beta$.

Lemma 3.3. Let $P \subseteq H(\kappa)$ and let $\left\langle\mathcal{P}_{\alpha}: \alpha \leq \kappa\right\rangle$ be the finite support iteration with $P$-symmetric systems as side conditions based on $\left\langle\left(\dot{\mathcal{Q}}_{\alpha}, \dot{\mathcal{R}}_{\alpha}\right): \alpha<\kappa\right\rangle$. Let $\alpha \leq \beta \leq \kappa$. If $q=\left(F_{q}, \Delta_{q}\right) \in \mathcal{P}_{\alpha}, r=\left(F_{r}, \Delta_{r}\right) \in \mathcal{P}_{\beta}$, and $q \leq\left._{\alpha} r\right|_{\alpha}$, then

$$
r \wedge_{\alpha} q:=\left(F_{q} \cup\left(F_{r} \uparrow[\alpha, \beta)\right), \Delta_{q} \cup \Delta_{r}\right),
$$

is a condition in $\mathcal{P}_{\beta}$ extending $r$.

\footnotetext{
${ }^{12}$ Note that $\beta_{i}$ is always less than $\kappa$ (even when $\left.\alpha=\kappa\right)$.
} 
Proof. The proof is a mechanical verification and proceeds by induction on $\beta \geq \alpha$. The crucial point is the use of the markers $\beta_{i}$ in the definition of the forcing. New side conditions $\left(N_{i}, \beta_{i}\right)$ appearing in $\Delta_{q}$ may well have the property that $N_{i} \cap[\alpha, \beta) \neq \emptyset$, but they will not impose any problematic promises - coming from clause $(b 4)$ in the definition - on ordinals $\xi$ occurring in $\operatorname{dom}\left(F_{r} \uparrow[\alpha, \beta)\right)$. The reason is simply that $\beta_{i} \leq \alpha$. The details of the proof are as follows.

Note that the case $\beta=\alpha$ is obvious, so let us start by assuming that $\beta$ is the successor of $\sigma$ with $\sigma \geq \alpha$. Clearly, $r \wedge_{\alpha} q$ satisfies clauses $(b 0)$ and $(b 1)$ in the definition of $\mathcal{P}_{\sigma+1}$. By the inductive hypothesis we know that the restriction of $r \wedge_{\alpha} q$ to $\sigma$, that is,

$$
\left.\left(r \wedge_{\alpha} q\right)\right|_{\sigma}=\left(F_{q} \cup\left(F_{r} \uparrow[\alpha, \sigma)\right), \Delta_{q} \cup \Delta_{\left.r\right|_{\sigma}}\right),
$$

is a condition in $\mathcal{P}_{\sigma}$ extending $\left.r\right|_{\sigma}$. Therefore, $r \wedge_{\alpha} q$ also satisfies (b2). If $\sigma \notin$ $\operatorname{dom}\left(F_{r}\right)$, then $r \wedge_{\alpha} q$ is a condition in $\mathcal{P}_{\sigma+1}$, since clause $(b 4)$ is automatically satisfied. If $\sigma \in \operatorname{dom}\left(F_{r}\right)$, then $\left.\left(r \wedge_{\alpha} q\right)\right|_{\sigma}$ forces in $\mathcal{P}_{\sigma}$ that $F_{r}(\sigma)$ is in $\dot{\mathcal{Q}}_{\sigma}$ (since $\left.r\right|_{\sigma}$ forces this and $\left.\left(r \wedge_{\alpha} q\right)\right|_{\sigma}$ extends $\left.\left.r\right|_{\sigma}\right)$. This concludes the verification of (b3) for $q \wedge_{\alpha} r$. Now we check that

$$
\left.\left(r \wedge_{\alpha} q\right)\right|_{\sigma} \Vdash_{\sigma}\left(N, F_{r}(\sigma)\right) \in \dot{\mathcal{R}}_{\sigma}
$$

for all those $N$ such that $(N, \sigma+1) \in \Delta_{q} \cup \Delta_{r}$ and $\sigma+1 \in N$. But such an $N$ is such that $(N, \sigma+1) \in \Delta_{r}$. Since $r$ satisfies $(b 4),\left.r\right|_{\sigma}$ (and hence, the restriction of $r \wedge_{\alpha} q$ to $\sigma)$ forces that $\left(N, F_{r}(\sigma)\right)$ is in $\dot{\mathcal{R}}_{\sigma}$. Finally note that the inductive hypothesis and the inclusion $\Delta_{r} \subseteq \Delta_{r \wedge_{\alpha} q}$ together imply that $r \wedge_{\alpha} q$ extends $r$. The case when $\beta$ is a nonzero limit ordinal follows directly from the inductive hypothesis.

Corollary 3.4. For every finite support iteration $\left\langle\mathcal{P}_{\alpha}: \alpha \leq \kappa\right\rangle$ with symmetric systems as side conditions and for all $\alpha<\beta \leq \kappa$, every maximal antichain in $\mathcal{P}_{\alpha}$ is a maximal antichain in $\mathcal{P}_{\beta}$, and therefore $\mathcal{P}_{\alpha}$ is a complete suborder of $\mathcal{P}_{\beta}$.

Lemma 3.5. Let $P \subseteq H(\kappa)$ and let $\left\langle\mathcal{P}_{\alpha}: \alpha \leq \kappa\right\rangle$ be the finite support iteration with $P$-symmetric systems as side conditions based on $\left\langle\left(\dot{\mathcal{Q}}_{\alpha}, \dot{\mathcal{R}}_{\alpha}\right): \alpha<\kappa\right\rangle$. Let $q_{1}=\left(F_{1}, \Delta_{1}\right)$ and $q_{2}=\left(F_{2}, \Delta_{2}\right)$ be conditions in $\mathcal{P}_{\alpha+1}$ such that there is a $\mathcal{P}_{\alpha}-$ name $\dot{x}$, a condition $r=\left(F_{r}, \Delta_{r}\right)$ in $\mathcal{P}_{\alpha}$, and a finite set $\left\{M_{j}: j \in n\right\}$ with the following properties:

(a) $\alpha+1 \leq \sup \left(M_{j} \cap \kappa\right)$ and $\left(M_{j}, \alpha\right) \in \Delta_{r}$ for all $j<n$,

(b) $r$ extends both $\left.q_{1}\right|_{\alpha}$ and $\left.q_{2}\right|_{\alpha}$,

(c) $\alpha \in \operatorname{dom}\left(F_{1}\right) \cap \operatorname{dom}\left(F_{2}\right)$ and $r$ forces in $\mathcal{P}_{\alpha}$ that $\dot{x}$ extends both $F_{1}(\alpha)$ and $F_{2}(\alpha)$ in $\dot{\mathcal{Q}}_{\alpha}$, and

(d) $r \Vdash_{\alpha}\left(M_{j}, \dot{x}\right) \in \dot{\mathcal{R}}_{\alpha}$ for all $j<n$ such that $\alpha \in M_{j}$.

Then,

$$
q_{3}=\left(F_{r} \cup\{\langle\alpha, \dot{x}\rangle\}, \Delta_{r} \cup \Delta_{1} \cup \Delta_{2} \cup\left\{\left(M_{j}, \alpha+1\right): j \in n\right\}\right)
$$

is a condition in $\mathcal{P}_{\alpha+1}$ extending both $q_{1}$ and $q_{2}$.

Proof. First we check that $q_{3}$ is in $\mathcal{P}_{\alpha+1}$. It follows from (a) and (b) that the restriction of $q_{3}$ to $\alpha$ is equal to $r$, and hence that $q_{3}$ satisfies clauses $(b 0)-(b 2)$. Condition (b3) for $q_{3}$ follows from (c). Finally we must show that $r$ forces $(N, \dot{x}) \in$ $\dot{\mathcal{R}}_{\alpha}$ for all those $N$ such that $(N, \alpha+1)$ is in $\Delta_{1} \cup \Delta_{2} \cup\left\{\left(M_{j}, \alpha+1\right): j \in n\right\}$ and such that $\alpha \in N$ (recall that if $(N, \gamma) \in \Delta_{r}$, then $\gamma \leq \alpha$ ). But for such an $N$, if $(N, \alpha+1) \in \Delta_{i}(i \in\{1,2\})$, it suffices to recall that $r \leq\left._{\alpha} q_{i}\right|_{\alpha}$, that $r$ forces $\dot{x} \leq_{\dot{Q}_{\alpha}} F_{i}(\alpha)$, and that (by clause (b4) applied to $\left.q_{i}\right)\left.q_{i}\right|_{\alpha}$ forces $\left(N, F_{i}(\alpha)\right) \in \dot{\mathcal{R}}_{\alpha}$. 
Hence, $r$ forces $(N, \dot{x}) \in \dot{\mathcal{R}}_{\alpha}$ by the downward closure of $\dot{\mathcal{R}}_{\alpha}$ with respect to $\dot{\mathcal{Q}}_{\alpha}$. The case when $(N, \alpha+1) \in\left\{\left(M_{j}, \alpha+1\right): j \in n\right\}$ follows from (d). Finally note that (b), (c) and the inclusion $\Delta_{i} \subseteq \Delta_{1} \cup \Delta_{2} \cup\left\{\left(M_{j}, \alpha+1\right): j \in n\right\}$ imply together that $q_{3}$ extends $q_{i}$ for $i \in\{1,2\}$.

Exactly the same proof establishes the following variant of Lemma 3.5.

Lemma 3.6. Let $P \subseteq H(\kappa)$ and let $\left\langle\mathcal{P}_{\alpha}: \alpha \leq \kappa\right\rangle$ be the finite support iteration with $P$-symmetric systems as side conditions based on $\left\langle\left(\dot{\mathcal{Q}}_{\alpha}, \dot{\mathcal{R}}_{\alpha}\right): \alpha<\kappa\right\rangle$. Let $q_{1}=\left(F_{1}, \Delta_{1}\right)$ and $q_{2}=\left(F_{2}, \Delta_{2}\right)$ be conditions in $\mathcal{P}_{\alpha+1}, r=\left(F_{r}, \Delta_{r}\right)$ a condition in $\mathcal{P}_{\alpha}$, and $\left\{M_{j}: j \in n\right\}$ a finite set with the following properties:

(a) $\alpha+1 \leq \sup \left(M_{j} \cap \kappa\right)$ and $\left(M_{j}, \alpha\right) \in \Delta_{r}$ for all $j<n$,

(b) $r$ extends both $\left.q_{1}\right|_{\alpha}$ and $\left.q_{2}\right|_{\alpha}$, and

(c) $\alpha \notin \operatorname{dom}\left(F_{1}\right) \cup \operatorname{dom}\left(F_{2}\right)$.

Then,

$$
q_{3}=\left(F_{r}, \Delta_{r} \cup \Delta_{1} \cup \Delta_{2} \cup\left\{\left(M_{j}, \alpha+1\right): j \in n\right\}\right)
$$

is a condition in $\mathcal{P}_{\alpha+1}$ extending both $q_{1}$ and $q_{2}$.

Suppose, in addition, that $\dot{x}$ is a $\mathcal{P}_{\alpha}$-name such that

(d) $r \Vdash_{\alpha}\left(M_{j}, \dot{x}\right) \in \dot{\mathcal{R}}_{\alpha}$ for all $j<n$ such that $\alpha \in M_{j}$, and

(e) $r \Vdash_{\alpha}(N, \dot{x}) \in \dot{\mathcal{R}}_{\alpha}$ for all $N$ such that $(N, \alpha+1) \in \Delta_{1} \cup \Delta_{2}$ and $\alpha \in N$.

Then,

$$
q_{3}^{\prime}=\left(F_{r} \cup\{\langle\alpha, \dot{x}\rangle\}, \Delta_{r} \cup \Delta_{1} \cup \Delta_{2} \cup\left\{\left(M_{j}, \alpha+1\right): j \in n\right\}\right)
$$

is a condition in $\mathcal{P}_{\alpha+1}$ extending both $q_{1}$ and $q_{2}$.

Lemma 3.7. Let $P \subseteq H(\kappa)$ and let $\left\langle\mathcal{P}_{\alpha}: \alpha \leq \kappa\right\rangle$ be the finite support iteration with $P$-symmetric systems as side conditions. Assume that $0 \leq \sigma<\alpha \leq \kappa$. Let $q_{\xi}=\left(F_{\xi}, \Delta_{\xi}\right)(\xi \in\{1,2\})$ be conditions in $\mathcal{P}_{\alpha}$ such that $\operatorname{supp}\left(q_{1}\right) \cup \operatorname{supp}\left(q_{2}\right) \subseteq \sigma$ and such that there exists a condition $r=\left(F_{r}, \Delta_{r}\right) \in \mathcal{P}_{\sigma}$ extending both $\left.q_{1}\right|_{\sigma}$ and $\left.q_{2}\right|_{\sigma}$. Then $q_{1}$ and $q_{2}$ are compatible in $\mathcal{P}_{\alpha}$.

Proof. Define $q_{3}=\left(F_{r}, \Delta_{r} \cup \Delta_{1} \cup \Delta_{2}\right)$. We prove by induction on $\beta, \sigma \leq \beta \leq \alpha$, that $\left.q_{3}\right|_{\beta}$ is a condition in $\mathcal{P}_{\beta}$ extending $\left.q_{1}\right|_{\beta}$ and $\left.q_{2}\right|_{\beta}$. The successor step follows from Lemma 3.6 .

Lemma 3.8. Let $P \subseteq H(\kappa)$ and let $\left\langle\mathcal{P}_{\alpha}: \alpha \leq \kappa\right\rangle$ be the finite support iteration with $P$-symmetric systems as side conditions based on $\left\langle\left(\dot{\mathcal{Q}}_{\alpha}, \dot{\mathcal{R}}_{\alpha}\right): \alpha<\kappa\right\rangle$. Let $0<\beta \leq \kappa$. Given conditions $q_{\xi}=\left(F_{\xi}, \Delta_{\xi}\right)(\xi \in\{0,1\})$ in $\mathcal{P}_{\beta}$, let $Z_{\xi}=\operatorname{supp}\left(q_{\xi}\right) \cup$ $\left(\beta \cap \bigcup \operatorname{dom}\left(\Delta_{q_{\xi}}\right)\right)$. Let $\alpha \leq \beta$ be such that $Z_{0} \cap Z_{1} \subseteq \alpha$, and assume there is a condition $r=\left(F_{r}, \Delta_{r}\right)$ in $\mathcal{P}_{\alpha}$ extending $\left.q_{0}\right|_{\alpha}$ and $\left.q_{1}\right|_{\alpha}$. Let $F_{r}^{0,1}=F_{r} \cup\left(F_{0} \uparrow\right.$ $[\alpha, \beta)) \cup\left(F_{1} \uparrow[\alpha, \beta)\right)$. Then the natural amalgamation of $r, q_{0}$ and $q_{1}$, i.e.,

$$
\left(q_{0} \wedge q_{1}\right) \wedge_{\alpha} r:=\left(F_{r}^{0,1}, \Delta_{r} \cup \Delta_{0} \cup \Delta_{1}\right),
$$

is a $\mathcal{P}_{\beta}$-condition extending $q_{0}$ and $q_{1}$.

Proof. The proof is by induction on $\beta \geq \alpha$. Note that the case $\beta=\alpha$ is clear, so let us start by assuming that $\beta$ is the successor of $\sigma$ with $\sigma \geq \alpha$. Clearly, $\left(q_{0} \wedge q_{1}\right) \wedge_{\alpha} r$ satisfies clauses $(b 0)$ and $(b 1)$. Using the inductive hypothesis we know that the restriction of the amalgamation to $\sigma$ is a condition in $\mathcal{P}_{\sigma}$ which extends both $\left.q_{0}\right|_{\sigma}$ and $\left.q_{1}\right|_{\sigma}$. In particular, if $\sigma \in \operatorname{dom}\left(F_{r}^{0,1}\right)$, then $\left.\left(\left(q_{0} \wedge q_{1}\right) \wedge_{\alpha} r\right)\right|_{\sigma}$ forces $F_{r}^{0,1}(\sigma) \in \dot{\mathcal{Q}}_{\sigma}$. Therefore, $\left(q_{0} \wedge q_{1}\right) \wedge_{\alpha} r$ also satisfies $(b 2)$ and (b3). Let us assume now that 
$\sigma \in \operatorname{dom}\left(F_{r}^{0,1}\right)$. In fact, since $\operatorname{supp}\left(q_{0}\right) \cap \operatorname{supp}\left(q_{1}\right) \subseteq \alpha$ and $\sigma \geq \alpha$, we may assume that $\sigma \in \operatorname{supp}\left(q_{0}\right) \backslash \operatorname{supp}\left(q_{1}\right)$ (the proof when $\sigma \in \operatorname{supp}\left(q_{1}\right)$ is identical). We must show that the condition $\left.\left(\left(q_{0} \wedge q_{1}\right) \wedge_{\alpha} r\right)\right|_{\sigma}$ forces $\left(N, F_{r}^{0,1}(\sigma)\right) \in \dot{\mathcal{R}}_{\sigma}$ for all those $N$ such that $(N, \sigma+1) \in \Delta_{0} \cup \Delta_{1}$ and $\sigma \in N$. Since $\sigma \geq \alpha$ and $Z_{0} \cap Z_{1} \subseteq \alpha$, it follows for such an $N$ that $(N, \sigma+1) \in \Delta_{0}$. Since $q_{0}$ satisfies $(b 4)$ and $F_{r}^{0,1}(\sigma)=F_{0}(\sigma)$, $\left.q_{0}\right|_{\sigma}$ (and hence, $\left.\left.\left(\left(q_{0} \wedge q_{1}\right) \wedge_{\alpha} r\right)\right|_{\sigma}\right)$ forces $\left(N, F_{r}^{0,1}(\sigma)\right) \in \dot{\mathcal{R}}_{\sigma}$. Finally note that the inductive hypothesis, the choice of $F_{r}^{0,1}$ and the inclusion $\Delta_{0} \cup \Delta_{1} \subseteq \Delta_{\left(q_{0} \wedge q_{1}\right) \wedge_{\alpha} r}$ together imply that $\left(q_{0} \wedge q_{1}\right) \wedge_{\alpha} r$ extends $q_{0}$ and $q_{1}$. The case when $\beta$ is a nonzero limit ordinal follows directly from the inductive hypothesis.

The final result in this section applies, assuming $\mathrm{CH}$, to iterations with $P$ symmetric systems as side conditions for $P$ for which there is a bijection $\varphi$ : $H(\kappa) \longrightarrow \kappa$ definable in $(H(\kappa), \in, P)$ and such that, moreover, each $\dot{\mathcal{Q}}_{\alpha}$ is forced to be a poset on $\omega_{1}^{V}$. It shows that all such iterations have the $\aleph_{2}$-chain condition. We will actually show that these forcings are $\aleph_{2}-$ Knaster 13

Lemma $3.9(\mathrm{CH})$. Let $\left\langle\mathcal{P}_{\alpha}: \alpha \leq \kappa\right\rangle$ be the finite support iteration with $P_{-}$ symmetric systems as side conditions based on $\left\langle\left(\dot{\mathcal{Q}}_{\alpha}, \dot{\mathcal{R}}_{\alpha}\right): \alpha<\kappa\right\rangle$. Suppose that

- there is a bijection $\varphi: H(\kappa) \longrightarrow \kappa$ definable in $(H(\kappa), \in, P)$, and that

- for all $\alpha<\kappa, \mathcal{P}_{\alpha}$ forces $\dot{\mathcal{Q}}_{\alpha} \subseteq \omega_{1}^{V}$.

Then for every ordinal $\alpha \leq \kappa, \mathcal{P}_{\alpha}$ is $\aleph_{2}-$ Knaster.

Proof. The proof is by induction on $\alpha$ and involves standard $\Delta$-system and pigeonhole principle arguments. The conclusion for $\alpha=0$ follows from $\mathrm{CH}$ : Suppose $m<\omega$ and $q_{\xi}=\left\{N_{i}^{\xi}: i<m\right\}$ is a $\mathcal{P}_{0}$-condition for each $\xi<\omega_{2}$. For notational convenience we are identifying a $\mathcal{P}_{0}$-condition $q$ with $\operatorname{dom}\left(\Delta_{q}\right)$, which is fine for this proof. By $\mathrm{CH}$ we may assume that $\left\{\bigcup_{i<m} N_{i}^{\xi}: \xi<\omega_{2}\right\}$ forms a $\Delta$-system with root $X$. Furthermore, by $\mathrm{CH}$ we may assume, for all $\xi, \xi^{\prime}<\omega_{2}$, that the structures $\left\langle\bigcup_{i<m} N_{i}^{\xi}, \in, P, X, N_{i}^{\xi}\right\rangle_{i<m}$ and $\left\langle\bigcup_{i<m} N_{i}^{\xi^{\prime}}, \in, P, X, N_{i}^{\xi^{\prime}}\right\rangle_{i<m}$ are isomorphic and that the corresponding isomorphism fixes $X$. The first assertion follows from the fact that there are only $\aleph_{1}$-many isomorphism types for such structures. For the second assertion note that, if $\Psi$ is the unique isomorphism between $\left\langle\bigcup_{i<m} N_{i}^{\xi}, \in, P, X, N_{i}^{\xi}\right\rangle_{i<m}$ and $\left\langle\bigcup_{i<m} N_{i}^{\xi^{\prime}}, \in, P, X, N_{i}^{\xi^{\prime}}\right\rangle_{i<m}$, then the restriction of $\Psi$ to $X \cap \kappa$ has to be the identity on $X \cap \kappa$. Since there is a bijection $\varphi: H(\kappa) \longrightarrow \kappa$ definable in $(H(\kappa), \in, P)$, we have that $\Psi$ fixes $X$ if and only if it fixes $X \cap \kappa$. It follows that $\Psi$ fixes $X$. Hence, by Lemma 2.4 we have, for all $\xi, \xi^{\prime}<\omega_{2}$, that $q_{\xi} \cup q_{\xi^{\prime}}$ extends both $q_{\xi}$ and $q_{\xi^{\prime}}$.

For $\alpha=\sigma+1$, suppose $q_{\xi}$ is a $\mathcal{P}_{\sigma+1}$-condition for each $\xi<\omega_{2}$. Suppose, without loss of generality, that each $q_{\xi}$ is of the form $q_{\xi}=\left(F_{\xi}, \Delta_{\xi}\right)$ with $\sigma \in \operatorname{dom}\left(F_{\xi}\right)$ (the proof in the case that there are $\aleph_{2}-$ many $q_{\xi}$ of the form $(F, \Delta)$ with $\operatorname{dom}(F) \subseteq \sigma$ follows directly from Lemma 3.6). By extending $q_{\xi}$ if necessary, we may assume that each $F_{\xi}(\sigma)$ is the canonical $\mathcal{P}_{\sigma}$-name for an actual ordinal in $\omega_{1}$. We may also assume by our induction hypothesis that all $\left.q_{\xi}\right|_{\sigma}$ are pairwise compatible. Let $r_{\xi, \xi^{\prime}} \in \mathcal{P}_{\sigma}$ be a condition extending both $\left.q_{\xi}\right|_{\sigma}$ and $\left.q_{\xi^{\prime}}\right|_{\sigma}$ for all $\xi<\xi^{\prime}<\omega_{2}$. Now find a set $I \subseteq \omega_{2}$ of size $\aleph_{2}$ such that for all $\xi<\xi^{\prime}$ in $I, F_{\xi}(\sigma)=F_{\xi^{\prime}}(\sigma)$. By Lemma 3.5 it follows now, for all such $\xi, \xi^{\prime}$, that the natural amalgamation of $r_{\xi, \xi^{\prime}}, q_{\xi}$ and $q_{\xi^{\prime}}$ is a $\mathcal{P}_{\sigma+1}$-condition extending $q_{\xi}$ and $q_{\xi^{\prime}}$.

${ }^{13}$ A forcing $\mathbb{P}$ is $\mu$-Knaster if every subset of $\mathbb{P}$ of cardinality $\mu$ includes a subset of cardinality $\mu$ of pairwise compatible conditions. 
For $\alpha$ a nonzero limit ordinal, suppose $q_{\xi}$ is a $\mathcal{P}_{\alpha}$-condition for all $\xi<\omega_{2}$. Suppose first that $\operatorname{cf}(\alpha) \neq \omega_{2}$. There is then some $\sigma<\alpha$ such that $I=\left\{\xi<\omega_{2}\right.$ : $\left.\operatorname{supp}\left(q_{\xi}\right) \subseteq \sigma\right\}$ has size $\aleph_{2}$. By induction hypothesis there is some $I^{\prime} \subseteq I$ of size $\aleph_{2}$ such that all $\left.q_{\xi}\right|_{\sigma}$ (for $\xi \in I^{\prime}$ ) are pairwise compatible in $\mathcal{P}_{\sigma}$. But now it follows from Lemma 3.7 that $q_{\xi}$ and $q_{\xi^{\prime}}$ are compatible in $\mathcal{P}_{\alpha}$ for all $\xi<\xi^{\prime}$ in $I^{\prime}$.

Finally, suppose $c f(\alpha)=\omega_{2}$. For each $\xi<\omega_{2}$, let $Z_{\xi}$ be equal to the union of the sets $\operatorname{supp}\left(q_{\xi}\right)$ and $\alpha \cap \bigcup \operatorname{dom}\left(\Delta_{q_{\xi}}\right)$. By CH we may find $I \subseteq \omega_{2}$ of size $\aleph_{2}$ such that $\left\{Z_{\xi}: \xi \in I\right\}$ forms a $\Delta$-system with $\operatorname{root} X$.

Now let $\sigma<\alpha$ be such that $X \subseteq \sigma$ ( $\sigma$ exists by $\left.c f(\alpha) \geq \omega_{1}\right)$. By induction hypothesis we may assume that all $\left.q_{\xi}\right|_{\sigma}$ are pairwise compatible in $\mathcal{P}_{\sigma}$. For all $\xi<\xi^{\prime}$ in $I$ let $r_{\xi, \xi^{\prime}}$ be a condition in $\mathcal{P}_{\sigma}$ extending $\left.q_{\xi}\right|_{\sigma}$ and $\left.q_{\xi^{\prime}}\right|_{\sigma}$. From Lemma 3.8 it follows then, for all such $\xi, \xi^{\prime}$, that the natural amalgamation of $r_{\xi, \xi^{\prime}}, q_{\xi}$ and $q_{\xi^{\prime}}$ is a $\mathcal{P}_{\alpha}$-condition extending $q_{\xi}$ and $q_{\xi^{\prime}}$.

\section{Proving Theorem 1.3}

We will now proceed to the definition of the forcing $\mathcal{P}$ witnessing Theorem 1.3 , The proof of Theorem 1.3 will then be given in a sequence of lemmas.

For this section, assume $\mathrm{CH}$ holds and let us fix a cardinal $\kappa$ such that $\kappa^{\aleph_{1}}=\kappa$ and $2^{<\kappa}=\kappa$. Let $\Phi: \kappa \longrightarrow H(\kappa)$ be a surjection such that for every $x$ in $H(\kappa)$, $\Phi^{-1}(\{x\})$ is unbounded in $\kappa$. Also let $\triangleleft$ be a well-order of $H\left(\kappa^{+}\right)$in order type $2^{\kappa}$. The bookkeeping function $\Phi$ exists by $2^{<\kappa}=\kappa$, and $\triangleleft$ exists since $\left|H\left(\kappa^{+}\right)\right|=2^{\kappa}$.

Let $\left\langle\theta_{\alpha}: \alpha \leq \kappa\right\rangle$ be the strictly increasing sequence of regular cardinals defined as $\theta_{0}=\left|2^{\kappa}\right|^{+}$and $\theta_{\alpha}=\left|2^{\sup \left\{\theta_{\beta}: \beta \leq \alpha\right\}}\right|^{+}$if $\alpha>0$. For each $\alpha \leq \kappa$ let $\mathcal{M}_{\alpha}^{*}$ be the collection of all countable elementary substructures of $H\left(\theta_{\alpha}\right)$ containing $\Phi, \triangleleft$ and $\left\langle\theta_{\beta}: \beta<\alpha\right\rangle$. Also let $\mathcal{M}_{\alpha}=\left\{N^{*} \cap H(\kappa): N^{*} \in \mathcal{M}_{\alpha}^{*}\right\}$ and note that if $\alpha<\beta$, then $\mathcal{M}_{\alpha}^{*}$ belongs to all members of $\mathcal{M}_{\beta}^{*}$ containing the ordinal $\alpha$.

The forcing $\mathcal{P}$ witnessing Theorem 1.3 will be $\mathcal{P}_{\kappa}$, where the sequence $\left\langle\mathcal{P}_{\alpha}\right.$ : $\alpha \leq \kappa\rangle$ is the finite support iteration with $\Phi$-symmetric systems as side conditions based on a certain sequence $\left\langle\left(\dot{\mathcal{Q}}_{\alpha}, \dot{\mathcal{R}}_{\alpha}\right): \alpha<\kappa\right\rangle$ of pairs of names. Let $\alpha<\kappa$ be given and suppose $\mathcal{P}_{\alpha}$ has been defined.

In Definition 4.1, and throughout the rest of the paper, we will abuse notation slightly when writing things like $N[H]$ : Given a set $N$, a partial order $\mathbb{P}$ and a filter $H \subseteq \mathbb{P}, N[H]=\left\{\tau_{H}: \tau \in N\right.$ is a $\mathbb{P}$-name $\}$. Note that we are not requiring that $\mathbb{P}$ be in $N$, or even that $\mathbb{P} \cap N$ be a definable class in $N$.

Definition 4.1 (For $\alpha<\kappa$, in $V\left[G_{\alpha}\right]$, where $G_{\alpha}$ is a $\mathcal{P}_{\alpha}$-generic filter). Let $\mathcal{Q}$ be a forcing on $\omega_{1}^{V}$. We will say that $\mathcal{Q} i s \quad V$-finitely proper (with respect to $G_{\alpha}$ ) if and only if there exists a club $D \subseteq\left([H(\kappa)]^{\aleph_{0}}\right)^{V}$ in $V$ with the following property:

If $m<\omega$ and $\left\{N_{i}: i \in m\right\} \subseteq D$ is such that $\left\{\left(N_{i}, \alpha\right): i<m\right\} \subseteq \Delta_{u}$ for some $u \in G_{\alpha}$ and such that $\mathcal{Q} \in N_{i}\left[G_{\alpha}\right]$ for all $i$, then for every $\nu \in \bigcap\left\{N_{i} \cap \omega_{1}: i \in m\right\}$ there exists some $\nu^{*}$ such that $\nu^{*}$ extends $\nu$ in $\mathcal{Q}$ and is $\left(N_{i}\left[G_{\alpha}\right], \mathcal{Q}\right)$-generic for all $i$.

The definition of $\left(\dot{\mathcal{Q}}_{\alpha}, \dot{\mathcal{R}}_{\alpha}\right)$ is the following: $\left(\dot{\mathcal{Q}}_{\alpha}, \dot{\mathcal{R}}_{\alpha}\right)$ is the $\triangleleft$-least pair of $\mathcal{P}_{\alpha}$-names in $H\left(\kappa^{+}\right)$with the following properties:

(1) If $\Phi(\alpha)=\dot{\mathcal{Q}}$ is a $\mathcal{P}_{\alpha}$-name for a nontrivia 14 -finitely proper forcing on $\omega_{1}^{V}$, then $\dot{\mathcal{Q}}_{\alpha}=\dot{\mathcal{Q}} 15$

\footnotetext{
${ }^{14}$ Nontrivial in the sense that it has some condition different from 0 .

${ }^{15}$ In Lemma 4.4 we will see that $\mathcal{P}_{\alpha}$ preserves $\omega_{1}$.
} 
(2) If $\Phi(\alpha)$ is not a $\mathcal{P}_{\alpha}$-name for a nontrivial $V$-finitely proper forcing on $\omega_{1}^{V}$, then $\dot{\mathcal{Q}}_{\alpha}$ is a $\mathcal{P}_{\alpha}$-name for trivial forcing on $\{0\}$ (which is a $V$-finitely proper forcing on $\omega_{1}^{V}$ ).

(3) $\dot{\mathcal{R}}_{\alpha}$ is a $\mathcal{P}_{\alpha}$-name for the set of pairs $(N, \nu)$ such that

(a) $\nu \in \dot{\mathcal{Q}}_{\alpha}$, and

(b) if $N \in \mathcal{M}_{\alpha+1}$, then $\nu$ is $\left(N\left[\dot{G}_{\alpha}\right], \dot{\mathcal{Q}}_{\alpha}\right)$-generic.

Note that $\dot{\mathcal{R}}_{\alpha}$ is forced to be downward closed with respect to $\dot{\mathcal{Q}}_{\alpha}$, so the construction makes sense. Note also that if $\alpha<\beta \leq \kappa, N^{*} \in \mathcal{M}_{\beta}^{*}$ and $\alpha \in N^{*}$, then $\mathcal{P}_{\alpha} \in N^{*}$.

We are going to prove the relevant properties of the forcings $\mathcal{P}_{\alpha}$. Theorem 1.3 will follow immediately from them.

The hypotheses of Lemma 3.9 clearly apply to our construction. Hence, every $\mathcal{P}_{\alpha}$ is $\aleph_{2}-$ Knaster.

Lemma 4.2. For every $\alpha \leq \kappa$ and $q \in \mathcal{P}_{\alpha}$ there is an extension $q^{\prime}$ of $q$ such that $F_{q^{\prime}}(\xi)$ is a canonical $\mathcal{P}_{\xi}$-name for an ordinal in $\omega_{1}^{V}$ for every $\xi \in \operatorname{supp}\left(q^{\prime}\right)$.

The proof of Lemma 4.2 is a straightforward induction using the fact that conditions in $\mathcal{P}_{\alpha}$ have finite support.

Definition 4.3. Given $\alpha \leq \kappa$, a condition $q \in \mathcal{P}_{\alpha}$, and a countable elementary substructure $N \prec H(\kappa)$, we will say that $q$ is $\left(N, \mathcal{P}_{\alpha}\right)$-pre-generic in case

- $\alpha<\kappa$ and the pair $(N, \alpha)$ is in $\Delta_{q}$, or else

- $\alpha=\kappa$ and the pair $(N, \sup (N \cap \kappa))$ is in $\Delta_{q}$.

The properness of all $\mathcal{P}_{\alpha}$ is an immediate consequence of the following lemma.

Lemma 4.4. Suppose $\alpha \leq \kappa$ and $N^{*} \in \mathcal{M}_{\alpha}^{*}$. Let $N=N^{*} \cap H(\kappa)$. Then the following conditions hold:

(1) $)_{\alpha}$ For every $q \in N$ there is some $q^{\prime} \leq_{\alpha} q$ such that $q^{\prime}$ is $\left(N, \mathcal{P}_{\alpha}\right)$-pre-generic.

$(2)_{\alpha}$ If $\mathcal{P}_{\alpha} \in N^{*}$ and $q \in \mathcal{P}_{\alpha}$ is $\left(N, \mathcal{P}_{\alpha}\right)$-pre-generic, then $q$ is $\left(N^{*}, \mathcal{P}_{\alpha}\right)$-generic.

Proof. The proof will be by induction on $\alpha$. We start with the case $\alpha=0$. For simplicity we are going to identify a $\mathcal{P}_{0}$-condition $q$ with $\operatorname{dom}\left(\Delta_{q}\right)$. The proof of $(1)_{0}$ is trivial: It suffices to set $q^{\prime}=q \cup\{N\}$.

The proof of $(2)_{0}$ is also easy: Let $E$ be a dense subset of $\mathcal{P}_{0}$ in $N^{*}$. It suffices to show that there is some condition in $E \cap N^{*}$ compatible with $q$. Notice that $q \cap N^{*} \in \mathcal{P}_{0}$ by Lemma 2.3 (ii). Hence, we may find a condition $q^{\circ} \in E \cap N^{*}$ extending $q \cap N^{*}$. Now let

$$
q^{*}=q \cup\left\{\Psi_{N, \bar{N}}(M): M \in q^{\circ}, \bar{N} \in \operatorname{dom}\left(\Delta_{q}\right), \delta_{\bar{N}}=\delta_{N}\right\} .
$$

By Lemma 2.3 (iii) we have that $q^{*}$ is a condition in $\mathcal{P}_{0}$ extending both $q$ and $q^{\circ}$.

Let us proceed to the more substantial case $\alpha=\sigma+1$. We start by proving $(1)_{\alpha}$. Assume first that $\sigma \in \operatorname{dom}\left(F_{q}\right)$ and let $\nu=F_{q}(\sigma)$. By Lemma 4.2 we may assume that $\nu$ is the canonical name for an ordinal in $\omega_{1}$. By $(1)_{\sigma}$ we may also assume, by extending $\left.q\right|_{\sigma}$, that $\left.q\right|_{\sigma}$ is $\mathcal{P}_{\sigma}$-pre-generic for $N$. Let $\dot{D}$ be the $\triangleleft$-first $\mathcal{P}_{\sigma}$-name for a club $D$ of $\left([H(\kappa)]^{\aleph_{0}}\right)^{V}$ in $V$ such that $D$ witnesses the $V$-finite properness of $\dot{\mathcal{Q}}_{\sigma}$ and note that $\left.q\right|_{\sigma}$ forces $N \in \dot{D}$ since it forces $N^{*}\left[\dot{G}_{\sigma}\right] \cap V=N^{*}$ (which follows from $(2)_{\sigma}$ and from the fact that $\left.q\right|_{\sigma}$ is $\mathcal{P}_{\sigma}$-pre-generic for $N$ ). By the definition of $V$-finite properness and the fact that $(N, \sigma) \in \Delta_{\left.q\right|_{\sigma}}$, there is then some $z=\left(F_{z}, \Delta_{z}\right) \in \mathcal{P}_{\sigma}$ extending $\left.q\right|_{\sigma}$ and an ordinal $\nu^{*}$ such that $z$ forces that 
$\nu^{*}$ is an $\left(N\left[\dot{G}_{\sigma}\right], \dot{\mathcal{Q}}_{\sigma}\right)$-generic condition extending $\nu$. It suffices to define $q^{\prime}$ as the condition $\left(F_{z} \cup\left\{\left\langle\sigma, \nu^{*}\right\rangle\right\}, \Delta_{q} \cup \Delta_{z} \cup\{(N, \alpha)\}\right.$ ) (Lemma 3.5 ensures that $q^{\prime}$ is a condition extending $q)$.

The proof in the case that $q=(F, \Delta)$ with $\operatorname{dom}(F) \subseteq \sigma$ can be reduced to the previous case by the following claim.

Claim 4.5. If $q=(F, \Delta)$ and $\sigma \notin \operatorname{dom}(F)$, then we can find a condition $q^{\prime}=\left(F^{\prime}, \Delta^{\prime}\right)$ extending $q$ and such that $\sigma \in \operatorname{dom}\left(F^{\prime}\right)$.

Proof. This is true, using Lemma 3.6. by essentially the same argument as above since $(2)_{\sigma}$ guarantees that $\left.q\right|_{\sigma}$ is also $\left(M^{*}, \mathcal{P}_{\sigma}\right)$-generic for all $M^{*} \in \mathcal{M}_{\sigma+1}^{*}$ such that $(M, \sigma+1) \in \Delta_{q}$ for $M=M^{*} \cap H(\kappa)$, which implies that a condition forcing that all these $M$ are in $\dot{D}$ can be found as in that argument.

Now let us prove $(2)_{\alpha}$. Let $E$ be an open dense set of $\mathcal{P}_{\alpha}$ in $N^{*}$. We should find a condition $\tilde{q} \in E \cap N^{*}$ compatible with $q$. Since $E$ is dense and open, we may start assuming that $q \in E$. By Claim 4.5 we may also assume that $\sigma \in \operatorname{dom}\left(F_{q}\right)$. Let $\nu=F_{q}(\sigma)$. Let $G_{\sigma}$ be a $\mathcal{P}_{\sigma}$-generic filter over $V$ with $\left.q\right|_{\sigma} \in G_{\sigma}$. By $(2)_{\sigma}$ we have that $G_{\sigma}$ is also generic over $N^{*}$. Define $E / G_{\sigma}$ as the set of those conditions in $E$ whose restriction to $\sigma$ belongs to $G_{\sigma}$, and $\tilde{E}$ as the set of those $\eta<\omega_{1}$ such that either

(i) there exists some $t \in E / G_{\sigma}$ such that $\sigma \in \operatorname{dom}\left(F_{t}\right)$ and $\eta=F_{t}(\sigma)$, or else

(ii) there is no $\eta^{\prime}$ in $\left(\dot{\mathcal{Q}}_{\sigma}\right)_{G_{\sigma}}$ extending $\eta$ for which there is any $t \in E / G_{\sigma}$ such that $\sigma \in \operatorname{dom}\left(F_{t}\right)$ and $\eta^{\prime}=F_{t}(\sigma)$.

Note that $\tilde{E}$ is a dense subset of $\left(\dot{\mathcal{Q}}_{\sigma}\right)_{G_{\sigma}}$ and that $\tilde{E} \in N^{*}\left[G_{\sigma}\right]$. In fact, $\tilde{E}$ is in $N\left[G_{\sigma}\right]$ by the $\aleph_{2}$-c.c. of $\mathcal{P}_{\sigma}$ and the fact that $\dot{\mathcal{R}}_{\sigma}$ is a partial order on $\omega_{1}^{V}$. Hence, by condition (b4) in the definition of $\mathcal{P}_{\alpha}$ together with the choice of $\dot{\mathcal{R}}_{\sigma}$, we know that there is some $\eta \in \tilde{E} \cap N\left[G_{\sigma}\right]$ such that $\nu$ and $\eta$ are $\left(\dot{\mathcal{Q}}_{\sigma}\right)_{G_{\sigma}}$-compatible.

Claim 4.6. Condition (i) above holds for $\eta$.

Proof. Let $r$ be a condition in $G_{\sigma}$ extending $\left.q\right|_{\sigma}$ and let $\eta^{\prime}$ be such that $r$ forces that $\eta^{\prime}$ is a condition in $\dot{\mathcal{Q}}_{\sigma}$ extending both $\eta$ and $\nu$. But then $q^{*}:=\left(F_{r} \cup\left\{\left\langle\sigma, \eta^{\prime}\right\rangle\right\rangle\right\}, \Delta_{r} \cup$ $\Delta_{q}$ ) is a $\mathcal{P}_{\alpha}$ condition extending $q$ by Lemma 3.5. $q^{*} \in E / G_{\sigma}$, and $\left.q^{*}\right|_{\sigma}$ forces that condition (i) holds for $\eta$ since $\eta^{\prime}$ witnesses the failure of (ii) for $\eta$. This shows that $\left.q\right|_{\sigma}$ forces that condition (i) holds for $\eta$.

By the above claim and by $N^{*}\left[G_{\sigma}\right] \prec H\left(\theta_{\sigma}\right)^{V}\left[G_{\sigma}\right]$, there is a condition $\tilde{q}$ in $E / G_{\sigma} \cap N^{*}\left[G_{\sigma}\right]$ such that $\sigma \in \operatorname{dom}\left(F_{\tilde{q}}\right)$ and $F_{\tilde{q}}(\sigma)=\eta$, and of course $\tilde{q} \in N$ since $N^{*}\left[G_{\sigma}\right] \cap V=N^{*}$ by $(2)_{\sigma}$. It remains to see that $\tilde{q}$ is compatible with $q$. For this, notice that there is some $w \in G_{\sigma}$ extending $\left.q\right|_{\sigma}$ and $\left.\tilde{q}\right|_{\sigma}$ and there is some $\eta^{*}<\omega_{1}$ such that $w$ forces that $\eta^{*}$ extends $\eta$ and $\nu$ in $\left(\dot{\mathcal{Q}}_{\sigma}\right)_{G_{\sigma}}$. But then $w$ forces that $\eta^{*}$ is $\left(M^{*}\left[\dot{G}_{\sigma}\right],\left(\dot{\mathcal{Q}}_{\sigma}\right)_{G_{\sigma}}\right)$-generic whenever $M^{*} \in \mathcal{M}_{\alpha}^{*}$ is such that $\left(M^{*} \cap H(\kappa), \alpha\right) \in$ $\Delta_{\tilde{q}} \cup \Delta_{q}$ since $\eta^{*}$ extends $\eta$ and $\nu$. It follows that $\left(F_{w} \cup\left\{\left\langle\sigma, \eta^{*}\right\rangle\right\}, \Delta_{q} \cup \Delta_{\tilde{q}} \cup \Delta_{w}\right)$ is a common extension of $q$ and $\tilde{q}$ by Lemma 3.5 .

It remains to prove the lemma for the case when $\alpha$ is a nonzero limit ordinal. We start out proving $(1)_{\alpha}$ : Let $\sigma \in \alpha \cap N$ be a bound for $\operatorname{supp}(q)$. By $(1)_{\sigma}$ there is a condition $t \leq\left._{\sigma} q\right|_{\sigma}$ which is pre-generic for $N$. Now let $q^{\prime}=\left(F_{t}, \Delta_{t} \cup \Delta_{q} \cup\right.$ $\{(M, \alpha \cap \sup (N \cap \kappa))\})$. It suffices to prove by induction on $\xi \in[\sigma, \alpha]$ that $\left.q^{\prime}\right|_{\xi}$ is a condition in $\mathcal{P}_{\xi}$. The limit case of the induction follows immediately from the induction hypothesis, and the successor case follows trivially from the fact that 
$\operatorname{dom}\left(F_{t}\right) \subseteq \sigma$, and so condition $(b 4)$ in the definition of $\mathcal{P}_{\xi+1}$ does not apply at that stage for $\left.q^{\prime}\right|_{\xi+1}$.

For $(2)_{\alpha}$, let $E \subseteq \mathcal{P}_{\alpha}$ be dense and open, $E \in N^{*}$, and let $q$ satisfy the hypothesis of $(2)_{\alpha}$. We want to find a condition in $E \cap N^{*}$ compatible with $q$. We may assume that $q \in E$.

Suppose first that $c f(\alpha)=\omega$. In this case we may take $\sigma \in N^{*} \cap \alpha$ above $\operatorname{supp}(q)$. Let $G_{\sigma}$ be $\mathcal{P}_{\sigma}$-generic with $\left.q\right|_{\sigma} \in G_{\sigma}$. In $N^{*}\left[G_{\sigma}\right]$ it is true that there is a condition $q^{\circ} \in \mathcal{P}_{\alpha}$ such that

(a) $q^{\circ} \in E$ and $\left.q^{\circ}\right|_{\sigma} \in G_{\sigma}$, and

(b) $\operatorname{supp}\left(q^{\circ}\right) \subseteq \sigma$

(the existence of such a $q^{\circ}$ is witnessed in $V\left[G_{\sigma}\right]$ by $q$ ).

Since $\left.q\right|_{\sigma}$ is $\left(N^{*}, \mathcal{P}_{\sigma}\right)$-generic by induction hypothesis, $q^{\circ} \in N^{*}$. By extending $q$ below $\sigma$ if necessary, we may assume that $\left.q\right|_{\sigma}$ decides $q^{\circ}$ and extends $\left.q^{\circ}\right|_{\sigma}$. But now, the natural amalgamation $\left(F_{q}, \Delta_{q} \cup \Delta_{q^{\circ}}\right)$ of $q$ and $q^{\circ}$ is a $\mathcal{P}_{\alpha}$-condition extending them by Lemma 3.7 .

Finally, suppose $c f(\alpha) \geq \omega_{1}$. We may assume that $\operatorname{supp}(q)$ is not bounded by $\sup (N \cap \alpha)$, as otherwise we can argue as in the $c f(\alpha)=\omega$ case. Thanks to Lemma 4.2, by extending $q$ if necessary we may also assume that, for every $\xi \in \operatorname{supp}(q)$, $F_{q}(\xi)$ is the canonical $\mathcal{P}_{\xi}$-name for some ordinal in $\omega_{1}$.

Notice that if $N^{\prime} \in \operatorname{dom}\left(\Delta_{q}\right)$ and $\delta_{N^{\prime}}<\delta_{N}$, then

$$
\sup \left(N^{\prime} \cap N \cap \alpha\right) \leq \sup \left(\Psi_{\bar{N}}, N\left(N^{\prime}\right) \cap \alpha\right) \in N \cap \alpha
$$

whenever $\bar{N} \in \operatorname{dom}\left(\Delta_{q}\right)$ is such that $\delta_{\bar{N}}=\delta_{N}$ and $N^{\prime} \in \bar{N}$. To see this, recall that $\Psi_{\bar{N}}, N$ fixes $\bar{N} \cap N \cap \kappa$. Also, $\sup \left(\Psi_{\bar{N}}, N\left(N^{\prime}\right) \cap \alpha\right) \in N \cap \alpha$ since in $N$ it holds that $\Psi_{\bar{N}}, N\left(N^{\prime}\right)$ is countable and that $\alpha$ has uncountable cofinality. This is the only place in the proof where the symmetry of the systems $\operatorname{dom}\left(\Delta_{q}\right)$ is needed. The symmetry of the systems $\operatorname{dom}\left(\Delta_{q}\right)$ is needed precisely to derive the conclusion that $\sup \left(N^{\prime} \cap N \cap \alpha\right)<\sup (N \cap \alpha)$ for every $N^{\prime} \in \operatorname{dom}\left(\Delta_{q}\right)$ with $\delta_{N^{\prime}}<\delta_{N}$.

Hence we may fix $\sigma \in N \cap \alpha$ such that:

(i) $\sup \left(N^{\prime} \cap N \cap \alpha\right)<\sigma$ for all $N^{\prime} \in \operatorname{dom}\left(\Delta_{q}\right)$ with $\delta_{N^{\prime}}<\delta_{N}$, and

(ii) if $\eta \in \operatorname{supp}(q)$ and $\eta<\sup (\alpha \cap N)$, then $\eta<\sigma$.

As in the case $\operatorname{cf}(\alpha)=\omega$, if $G_{\sigma}$ is $\mathcal{P}_{\sigma}$-generic with $\left.q\right|_{\sigma} \in G_{\sigma}$, then in $N^{*}\left[G_{\sigma}\right]$ we can find a condition $q^{\circ} \in \mathcal{P}_{\alpha}$ such that $q^{\circ} \in E,\left.q^{\circ}\right|_{\sigma} \in G_{\sigma}, \operatorname{supp}\left(q^{\circ}\right) \backslash \sigma \neq \emptyset$ and such that, for each $\xi \in \operatorname{supp}\left(q^{\circ}\right), F_{q^{\circ}}(\xi)$ is the canonical $\mathcal{P}_{\xi}$-name for an ordinal in $\omega_{1}$ (again, the existence of such a condition is witnessed in $V\left[G_{\sigma}\right]$ by $q$ ), and such a $q^{\circ}$ will necessarily be in $N^{*}$. By extending $q$ below $\sigma$ we may assume that $\left.q\right|_{\sigma}$ decides $q^{\circ}$ and extends $\left.q^{\circ}\right|_{\sigma}$. The proof of $(2)_{\alpha}$ in this case will be finished if we can show that there is a condition $q^{\dagger}$ extending $q$ and $q^{\circ}$. The condition $q^{\dagger}$ can be built by recursion on $\operatorname{supp}\left(q^{\circ}\right) \backslash \sigma$ (note that by the choice of $\sigma$, $\min (\operatorname{supp}(q) \backslash \sigma) \geq \sup (N \cap \alpha)$, and therefore $\left.\min (\operatorname{supp}(q) \backslash \sigma)>\max \left(\operatorname{supp}\left(q^{\circ}\right)\right)\right)$. This finite construction mimics the proof of $(1)_{\beta}$ for successor $\beta$, but also uses the assumption of $V$-finite properness. The details are as follows.

Let $\left(\xi_{i}\right)_{i<r}$ be the strictly increasing enumeration of $\operatorname{supp}\left(q^{\circ}\right) \backslash \sigma$. Note that $r>0$, so $r-1 \geq 0$. We build a sequence $\left(q_{i}\right)_{i<r}$ of conditions as follows:

For $i=0$, we first extend $\left.q\right|_{\sigma}$ to a $\mathcal{P}_{\xi_{0}}$-condition $\bar{q}$ extending $\left.q\right|_{\xi_{0}}$ and $\left.q^{\circ}\right|_{\xi_{0}} \cdot \bar{q}$ can be found by appealing to Lemma 3.7 if $\sigma<\xi_{0}$, and if $\sigma=\xi_{0}$ it is enough of course to set $q_{0}=\left.q\right|_{\sigma}$. Now note that $F_{q^{\circ}}\left(\xi_{0}\right)=\check{\pi}$ is the canonical name for an ordinal $\pi$ in the intersection of all $\bar{N}$ with $\bar{N} \in \operatorname{dom}\left(\Delta_{q}\right), \xi_{0} \in \bar{N}$ and $\delta_{\bar{N}} \geq \delta_{N^{*}}$ 
(on the other hand, (i) implies that there is no $N^{\prime} \in \operatorname{dom}\left(\Delta_{q}\right)$ with $\delta_{N^{\prime}}<\delta_{N}$ such that $\xi_{0} \in N^{\prime}$ ). Hence, since $\dot{\mathcal{Q}}_{\xi_{0}}$ is a $\mathcal{P}_{\xi_{0}}$-name for a $V$-finitely proper poset on $\omega_{1}$, there is an ordinal $\pi^{*}$ and an extension $r$ of $\bar{q}$ forcing that $\pi^{*}$ extends $\pi$ in $\dot{\mathcal{Q}}_{\xi_{0}}$ and is $\left(\bar{N}\left[\dot{G}_{\xi_{0}}\right], \dot{\mathcal{Q}}_{\xi_{0}}\right)$-generic for all relevant $\bar{N}$ (i.e., such that there some $\beta$ such that $(\bar{N}, \beta) \in \Delta_{q}, \beta \geq \xi_{0}+1$ and $\left.\bar{N} \in \mathcal{M}_{\xi_{0}+1}\right)$. The reason is that $\bar{q}$ forces that there is a club $\dot{D}$ witnessing the $V$-finite properness of $\dot{\mathcal{Q}}_{\xi_{0}}$, and such that every relevant $\bar{N}$ is in $\dot{D}$. This $\dot{D}$ can be taken to be the first club, in the well-order of $H\left(\kappa^{+}\right)\left[\dot{G}_{\xi_{0}}\right]$ induced by $\triangleleft$, witnessing the $V$-finite properness of $\dot{\mathcal{Q}}_{\xi_{0}}$. (For such a relevant $\bar{N}$ there is some $\bar{N}^{*} \in \mathcal{M}_{\xi_{0}+1}^{*}$ containing $\triangleleft$ and such that $\bar{N}=\bar{N}^{*} \cap H(\kappa)$ which, since $\triangleleft \in \bar{N}^{*}$, implies that $\bar{N}^{*}$ contains a name for $\dot{D}$. Applying this fact and $(2)_{\xi_{0}}$ we conclude that $\bar{q}$ forces $\bar{N} \in \dot{D}$.) It follows now from Lemma 3.5 that there is a $\mathcal{P}_{\xi_{0}+1}$-condition $q_{0}$ extending $r,\left.q\right|_{\xi_{0}+1}$ and $\left.q^{\circ}\right|_{\xi_{0}+1}$.

For $i$ such that $i+1<r$, we assume inductively that $q_{i} \in \mathcal{P}_{\xi_{i}+1}$ extends $\left.q\right|_{\xi_{i}+1}$ and $\left.q^{\circ}\right|_{\xi_{i}+1}$, and obtain $q_{i+1} \in \mathcal{P}_{\xi_{i+1}+1}$ from $q_{i}$ by arguing exactly as in the case $i=0$ with $\xi_{i+1}$ instead of $\xi_{0}$ and starting with $q_{i}$ rather than $\left.q\right|_{\sigma}$. In the end we obtain $q_{i+1} \in \mathcal{P}_{\xi_{i+1}+1}$ extending both $\left.q\right|_{\xi_{i+1}+1}$ and $\left.q^{\circ}\right|_{\xi_{i+1}+1}$.

Let $\mu=\xi_{r-1}=\max \left(\operatorname{supp}\left(q^{\circ}\right)\right)$ and let

$$
q^{\dagger}=\left(F_{q_{r-1}} \cup\left(F_{q} \uparrow[\mu+1, \alpha)\right), \Delta_{q_{r-1}} \cup \Delta_{q^{\circ}} \cup \Delta_{q}\right) .
$$

Claim 4.7. $q^{\dagger}$ is a condition in $\mathcal{P}_{\alpha}$ extending both $q$ and $q^{\circ}$.

Proof. We prove by induction that if $\mu+1 \leq \xi \leq \alpha$, then $\left.q^{\dagger}\right|_{\xi}$ is in $\mathcal{P}_{\xi}$ and $\left.q^{\dagger}\right|_{\xi} \leq_{\xi}$ $\left.q^{\circ}\right|_{\xi},\left.q\right|_{\xi}$. Note that the case $\xi=\mu+1$ follows from the fact that $q_{r-1} \leq\left._{\mu+1} q^{\circ}\right|_{\mu+1}$, $\left.q\right|_{\mu+1}$ and $\left.q^{\dagger}\right|_{\mu+1}=q_{r-1}$. Assume now that $\xi$ is the successor of an ordinal $\eta \geq \mu+1$. We show that $\left.q^{\dagger}\right|_{\eta+1}$ satisfies clause (b4) in the definition of $\mathcal{P}_{\eta+1}$ (clearly it satisfies the other clauses). In other words, we must show that if $\eta \in \operatorname{dom}\left(F_{q}\right)$, then $\left.q^{\dagger}\right|_{\eta}$ forces that $F_{q}(\eta)$ is $\left(M\left[\dot{G}_{\eta}\right], \dot{\mathcal{Q}}_{\eta}\right)$-generic for all those $M \in \mathcal{M}_{\eta+1}$ for which there exists an ordinal $\beta \geq \eta+1$ such that $(M, \beta) \in \Delta_{q_{r-1}} \cup \Delta_{q^{\circ}} \cup \Delta_{q}$. But such a pair $(M, \beta)$ cannot be in $\Delta_{q_{r-1}}$, since all markers occurring in side conditions in $q_{r-1} \in \mathcal{P}_{\mu+1}$ are at most $\mu+1<\eta+1$. On the other hand, (ii) implies that $\eta \in \operatorname{supp}(q) \backslash \sigma=\operatorname{supp}(q) \backslash(N \cap \alpha)$. So, there is no $M \in \operatorname{dom}\left(\Delta_{q^{\circ}}\right)$ such that $M \in \mathcal{M}_{\eta+1}$ (such a countable $M$ is in $N$, and therefore $M \cap \alpha \subseteq N \cap \alpha$ ), and hence $(M, \beta)$ is not in $\Delta_{q^{\circ}}$. We conclude that such a pair $(M, \beta)$ is in $\Delta_{q}$. By $(b 4)$ applied to $\left.q\right|_{\eta+1}$, we have that $\left.q\right|_{\eta}$ (and hence, $\left.q^{\dagger}\right|_{\eta}$ ) forces what we want. Finally note that the inductive hypothesis $\left.q^{\dagger}\right|_{\eta} \leq\left._{\eta} q^{\circ}\right|_{\eta},\left.q\right|_{\eta}$, the definition of $q^{\dagger}$, and the fact that the maximum of the support of $q^{\circ}$ is equal to $\mu<\eta$ together imply that $\left.q^{\dagger}\right|_{\eta+1} \leq\left._{\eta+1} q^{\circ}\right|_{\eta+1},\left.q\right|_{\eta+1}$. The case when $\xi$ is a limit follows from the inductive hypothesis.

The above claim finishes the proof of $(2)_{\alpha}$ in the present case and the proof of the lemma.

Corollary 4.8. For every $\alpha \leq \kappa, \mathcal{P}_{\alpha}$ is proper.

Given an ordinal $\alpha<\kappa$, we let $\dot{G}_{\alpha}^{+}$be a $\mathcal{P}_{\alpha+1}$-name for the collection of all $\nu$ for which there exists a condition $q \in \dot{G}_{\alpha+1}$ with $\alpha \in \operatorname{dom}\left(F_{q}\right)$ and $F_{q}(\alpha)=\nu$.

The following lemmas are easy.

Lemma 4.9. If $\alpha<\kappa$, then $\mathcal{P}_{\alpha+1}$ forces that $\dot{G}_{\alpha}^{+}$generates a $V\left[\dot{G}_{\alpha}\right]$-generic filter over $\dot{\mathcal{Q}}_{\alpha}$. 
Proof. It is easy to see that $\dot{G}_{\alpha}^{+}$is forced to be a set of pairwise compatible $\dot{\mathcal{Q}}_{\alpha}-$ conditions, so it suffices to show that $\mathcal{P}_{\alpha+1}$ forces $\dot{G}_{\alpha}^{+} \cap D \neq \emptyset$ for every dense subset $D$ of $\dot{\mathcal{Q}}_{\alpha}$ in $V\left[\dot{G}_{\alpha}\right]$. For this, note that if $\dot{D}$ is a $\mathcal{P}_{\alpha}-$ name for a dense subset of $\dot{\mathcal{Q}}_{\alpha}$, then a consecutive application of Claim 4.5 and Lemma 3.5 shows that the set of $q \in \mathcal{P}_{\alpha+1}$ with $\alpha \in \operatorname{supp}(q)$ and such that $\left.q\right|_{\alpha}$ forces that $F_{q}(\alpha)$ is in $\dot{D}$ is a dense subset of $\mathcal{P}_{\alpha+1}$.

Lemma 4.10. $\mathcal{P}_{\kappa}$ forces $2^{\aleph_{0}}=\kappa$.

Proof. The inequality $2^{\aleph_{0}} \geq \kappa$ follows for example from the fact that there are $\kappa$-many ordinals $\alpha<\kappa$ such that $\Phi(\alpha)$ is Cohen forcing, since Cohen forcing has the c.c.c.

The inequality $2^{\aleph_{0}} \leq \kappa$ follows from the fact that, by Lemma 3.9 together with $\kappa^{\aleph_{1}}=\kappa$, there are exactly $\kappa$-many nice $\mathcal{P}_{\kappa}$-names for subsets of $\omega$ (see for example 11] for a discussion of nice names and arguments involving counting of nice names).

We are ready to prove our main theorem.

Proof of Theorem 1.3. As we said, our forcing will be $\mathcal{P}_{\kappa}$. By Lemma 3.9, Corollary 4.8 and Lemma 4.10, it suffices to show that $\mathcal{P}_{\kappa}$ forces $\mathrm{PFA}^{\text {fin }}\left(\omega_{1}\right)$. But this follows easily from the following claim together with Lemma 4.9

Claim 4.11. If $\dot{\mathcal{Q}}$ is a $\mathcal{P}_{\kappa}$-name for a nontrivial finitely proper poset defined on $\omega_{1}$ and $\left(\dot{D}_{i}\right)_{i<\omega_{1}}$ is a sequence of $\mathcal{P}_{\kappa}$-names for dense subsets of $\dot{\mathcal{Q}}$, then there is a high enough $\alpha<\kappa$ such that $\dot{\mathcal{Q}}$ and all members of $\left(\dot{D}_{i}\right)_{i<\omega_{1}}$ are $\mathcal{P}_{\alpha}$-names and $\Phi(\alpha)=\dot{\mathcal{Q}}$ is a $\mathcal{P}_{\alpha}$-name for a $V$-finitely proper forcing with respect to $\dot{G}_{\alpha}$.

Proof. By the $\aleph_{2}$-chain condition of $\mathcal{P}_{\kappa}$, together with Corollary 3.4 and with the fact that the relevant information about $\dot{\mathcal{Q}}$ and $\left(\dot{D}_{i}\right)_{i<\omega_{1}}$ is decided by a collection of $\aleph_{1}$-many maximal antichains of $\mathcal{P}_{\kappa}$, there is some $\alpha<\kappa$ such that $\dot{\mathcal{Q}}$ and all $\dot{D}_{i}$ are $\mathcal{P}_{\alpha}$-names. Furthermore, by the choice of $\Phi$ we may assume $\Phi(\alpha)=\dot{\mathcal{Q}}$. Therefore we will be done if we show that $\mathcal{P}_{\alpha}$ forces that $\mathcal{Q}$ is $V$-finitely proper with respect to $\dot{G}_{\alpha}$. The witnessing club for this can be taken to be any club $D$ consisting of structures of the form $N^{*} \cap H(\kappa)^{V}$ where $N^{*} \in \mathcal{M}_{\kappa}^{*}$ and $\alpha \in N^{*}$.

Now let $q \in \mathcal{P}_{\alpha}$, let $\left\{N_{i}: i<m\right\} \subseteq D$ be a finite set such that $\left\{\left(N_{i}, \alpha\right): i<\right.$ $m\} \subseteq \Delta_{q}$, and let $\nu \in \bigcap_{i} N_{i} \cap \omega_{1}$. Then

$$
q^{*}:=\left(F_{q}, \Delta_{q} \cup\left\{\left(N_{i}, \sup \left(N_{i} \cap \kappa\right)\right): i<m\right\}\right)
$$

is clearly a condition in $\mathcal{P}_{\kappa}$ extending $q$ (viewing $q$ as a $\mathcal{P}_{\kappa}$-condition in the natural way). Let $G$ be any generic filter for $\mathcal{P}_{\kappa}$ containing $q^{*}$. For each $i$, since $N_{i}=$ $N^{*} \cap H(\kappa)^{V}$ for some $N^{*} \in \mathcal{M}_{\kappa}^{*}$ and $q^{*}$ is $\mathcal{P}_{\kappa}$-pre-generic for $N_{i}$, we have that $N_{i}[G] \cap V=N_{i}$ by Lemma 4.4. By finite properness of $\mathcal{Q}:=\dot{\mathcal{Q}}_{G}$ there is then some condition $\nu^{*}<\omega_{1}$ in $\mathcal{Q}$ extending $\nu$ and $\left(N_{i}[G], \mathcal{Q}\right)$-generic for all $i$. But since, for all $i<m, N_{i}[G] \cap \omega_{1}=N_{i} \cap \omega_{1}=N_{i}\left[G \cap \mathcal{P}_{\alpha}\right] \cap \omega_{1}$, it follows that $\nu^{*}$ is also $\left(N_{i}\left[G \cap \mathcal{P}_{\alpha}\right], \mathcal{Q}\right)$-generic for all such $i$. This finishes the proof since then, by Corollary [3.4, $q$ can be extended to a $\mathcal{P}_{\alpha}$-condition forcing that $\nu^{*}$ is $\left(N_{i}\left[G \cap \mathcal{P}_{\alpha}\right], \mathcal{Q}\right)$-generic for all $i<m$. 
5. Applichtions: PFA $\omega_{\omega_{1}}^{\text {FIN }}$ And the Club Filter on $\omega_{1}$

In this final section we show that $\mathrm{PFA}_{\omega_{1}}^{\text {fin }}$ implies both $\neg \mathrm{WCG}$ and $\neg \mho$. It will be convenient to introduce the following natural notion of rank of an ordinal with respect to a set of ordinals 16

Definition 5.1. Given a set $X$ and an ordinal $\delta$, we define the Cantor-Bendixson rank of $\delta$ with respect to $X, \operatorname{rank}(X, \delta)$, by specifying that

- $\operatorname{rank}(X, \delta) \geq 1$ if and only if $\delta$ is a limit point of ordinals in $X$.

- If $\mu>1, \operatorname{rank}(X, \delta) \geq \mu$ if and only if for every $\eta<\mu, \delta$ is a limit of ordinals $\epsilon$ with $\operatorname{rank}(X, \epsilon) \geq \eta$.

A function $F: \omega_{1} \longrightarrow \omega_{1}$ is normal if it is strictly increasing and continuous. The following two lemmas are easy consequences of our definition of rank.

Lemma 5.2. Let $A \subseteq X$ be sets of ordinals and let $\delta$ be an ordinal. If $\operatorname{rank}(A, \delta)<$ $\operatorname{rank}(X, \delta)$, then $\operatorname{rank}(X \backslash A, \delta)=\operatorname{rank}(X, \delta)$.

Lemma 5.3. Given any strictly increasing finite function $f \subseteq \omega_{1} \times \omega_{1}$, if $\operatorname{rank}(f(\xi)$, $f(\xi)) \geq \xi$ for every $\xi \in \operatorname{dom}(f)$, then $f$ can be extended to a normal function $F: \omega_{1} \longrightarrow \omega_{1}$.

Proof. It suffices to prove, for all $\xi<\omega_{1}$, that if $\xi_{0}<\xi$ and $\alpha<\beta<\omega_{1}$ are such that $\operatorname{rank}(\alpha, \alpha) \geq \xi_{0}$ and $\operatorname{rank}(\beta, \beta) \geq \xi$, then there is a strictly increasing and continuous function $h:\left[\xi_{0}, \xi\right] \longrightarrow[\alpha, \beta]$ with $h\left(\xi_{0}\right)=\alpha$ and $h(\xi)=\beta$. The proof of this fact is immediate by induction on $\xi$ and uses the definition of rank.

Let us first show the following.

Proposition 5.4. PFA $^{f i n}\left(\omega_{1}\right)$ implies $\neg \mathrm{WCG}$.

Proof. Let $\mathcal{A}=\left\langle A_{\delta}: \delta \in \operatorname{Lim}\left(\omega_{1}\right)\right\rangle$ be a ladder system on $\omega_{1}$. We want to show that there is a club $C \subseteq \omega_{1}$ such that $C \cap A_{\delta}$ is finite for every limit ordinal $\delta \in C$. Let $\mathbb{P}_{\mathcal{A}}$ be the following partial order:

A condition in $\mathbb{P}_{\mathcal{A}}$ is a pair $\left(f,\left\langle b_{\delta}: \delta \in D\right\rangle\right)$ with the following properties:

(1) $f \subseteq \omega_{1} \times \omega_{1}$ is a strictly increasing finite function such that $\operatorname{rank}(f(\xi), f(\xi))$ $\geq \xi$ for every $\xi \in \operatorname{dom}(f)$.

(2) $\bar{D} \subseteq \operatorname{dom}(f) \cap \operatorname{Lim}\left(\omega_{1}\right)$, and for each $\delta \in D, b_{\delta}$ is a finite subset of $A_{f(\delta)}$ and $\operatorname{range}(f) \cap A_{f(\delta)}=b_{\delta}$.

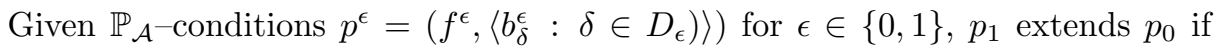
and only if

(i) $f^{0} \subseteq f^{1}$,

(ii) $D_{0} \subseteq D_{1}$, and

(iii) $b_{\delta}^{0}=b_{\delta}^{1}$ for every $\delta \in D_{0}$.

The forcing $\mathbb{P}_{\mathcal{A}}$ is a natural variation of Baumgartner's forcing for adding a club of $\omega_{1}$ with finite conditions (see [3]). It clearly has size $\aleph_{1}$, so in order to show that there is a club of $\omega_{1}$ avoiding $\mathcal{A}$ it will suffice to argue that $\mathbb{P}_{\mathcal{A}}$ adds such a club and that it is finitely proper. The proof of the following lemma is completely standard and essentially like in the corresponding proof for Baumgartner's forcing,

\footnotetext{
${ }^{16}$ This notion of rank will be particularly useful in the proof that $\mathrm{PFA}_{\omega_{1}}^{\mathrm{fin}}$ implies $\neg \mho$ (Proposition 5.8.
} 
using Lemma 5.3 and the fact that if $\left(f,\left\langle b_{\delta}: \delta \in D\right\rangle\right)$ is a condition in $\mathbb{P}_{\mathcal{A}}$ and $\delta \in D$, then $\operatorname{rank}\left(f(\delta) \backslash A_{f(\delta)}, f(\delta)\right) \geq \delta$ (which is true by Lemma 5.2, since $\left.\operatorname{rank}\left(A_{f(\delta)}, f(\delta)\right)=1<\delta\right)$.

Lemma 5.5. Let $p=\left(f,\left\langle b_{\delta}: \delta \in D\right\rangle\right) \in \mathbb{P}_{\mathcal{A}}$. Then the following is true:

(i) For all $\beta<\omega_{1}$ there is some $\left(f^{\prime},\left\langle b_{\delta}^{\prime}: \delta \in D^{\prime}\right\rangle\right)$ in $\mathbb{P}_{\mathcal{A}}$ extending $p$ and such that $\beta \in \operatorname{dom}\left(f^{\prime}\right)$. If $\beta$ is a limit ordinal, then we may take $D^{\prime}$ such that $\beta \in D^{\prime}$. Furthermore, if $\beta \notin \operatorname{dom}(f)$ is such that $f^{\prime \prime} \beta \subseteq \beta$ and $\operatorname{rank}(\beta, \beta)=\beta$, then we may take $f^{\prime}$ such that $f^{\prime}(\beta)=\beta$.

(ii) For every limit ordinal $\alpha \in \operatorname{dom}(f)$ and every $\xi<f(\alpha)$ there is some $\left(f^{\prime},\left\langle b_{\delta}^{\prime}: \delta \in D^{\prime}\right\rangle\right)$ in $\mathbb{P}_{\mathcal{A}}$ extending $p$ and there is some $\beta \in \operatorname{dom}\left(f^{\prime}\right) \cap \alpha$ such that $f^{\prime}(\beta)>\xi$.

It follows from Lemma 5.5 that $\mathbb{P}_{\mathcal{A}}$ forces that the union of the ranges of all first components of conditions in the generic filter is a club of $\omega_{1}^{V}$ and that this club has finite intersection with each $A_{\delta}$.

It remains to show that $\mathbb{P}_{\mathcal{A}}$ is finitely proper. The proof of this is basically the same as the proof that $\mathbb{P}_{\mathcal{A}}$ is proper (which is quite well known; see [21]). For completeness we give the proof of finite properness.

Lemma 5.6. $\mathbb{P}_{\mathcal{A}}$ is finitely proper.

Proof. Let $\left\{N_{i}: i \in m\right\}$ be a finite set of countable elementary substructures of $H\left(\omega_{2}\right)$ such that $\mathbb{P}_{\mathcal{A}} \in N_{i}$ for all $i$. Since $\operatorname{rank}\left(\delta_{N_{i}}, \delta_{N_{i}}\right)=\delta_{N_{i}}$ for all $i$, by Lemma 5.5 (i) we know that every condition in $\bigcap_{i<m} N_{i}$ can be extended to a condition $\left(f,\left\langle b_{\delta}: \delta \in D\right\rangle\right)$ such that $\delta_{N_{i}} \in \operatorname{dom}(f)$ and $f\left(\delta_{N_{i}}\right)=\delta_{N_{i}}$ for all $i$. Hence, it will suffice to show that if $p=\left(f,\left\langle b_{\delta}: \delta \in D\right\rangle\right)$ is a condition in $\mathbb{P}_{\mathcal{A}}$ and each $\delta_{N_{i}}$ is a fixed point of $f$, then $p$ is $\left(N_{i}, \mathbb{P}_{\mathcal{A}}\right)$-generic for all $i$. For this, fix $i<m, E$ a dense subset of $\mathbb{P}_{\mathcal{A}}$ in $N_{i}$, and suppose without loss of generality that $p$ is in $E$. We may also assume that $f\left\lceil\delta_{N_{i}}\right.$ is nonempty. It suffices to show that $p$ is compatible with a condition in $E \cap N_{i}$.

For this, let $\mu=\max \left(\operatorname{range}\left(f\left\lceil\delta_{N_{i}}\right)\right)\right.$ and let $g: \omega_{1} \backslash(\mu+1) \longrightarrow \omega_{1}$ be the function sending each $\nu$ in $\omega_{1} \backslash(\mu+1)$ to the least $\xi$ with the property that there is a condition $p^{\prime}$ in $E, p^{\prime}=\left(f^{\prime},\left\langle b_{\delta}^{\prime}: \delta \in D^{\prime}\right\rangle\right)$, such that

(a) $p^{\prime}$ extends $\left(f \uparrow \delta_{N_{i}},\left\langle b_{\delta}: \delta \in D \cap \delta_{N_{i}}\right\rangle\right)$,

(b) $f^{\prime} \uparrow \delta_{N_{i}}=f \uparrow \delta_{N_{i}}$,

(c) $\xi>\nu$, and

(d) $\xi$ is the least ordinal in the range of $f^{\prime}$ strictly above $\mu$.

Note that for every $\nu \in \delta_{N_{i}} \backslash(\mu+1), \delta_{N_{i}}$ and $p$ witness together that the set of pairs $\left(\xi, p^{\prime}\right)$ satisfying (a)-(d) is nonempty. Hence $g$ is a well-defined function. Note also that $g$, being definable from the condition $\left(f\left\lceil\delta_{N_{i}},\left\langle b_{\delta}: \delta \in D \cap \delta_{N_{i}}\right\rangle\right.\right.$ ), is in $N_{i}$ since $f\left(\delta_{N_{i}}\right)=\delta_{N_{i}}$, and therefore $\left(f\left\lceil\delta_{N_{i}},\left\langle b_{\delta}: \delta \in D \cap \delta_{N_{i}}\right\rangle\right)\right.$ is in $N_{i}$. It follows that the club $C$ of $\eta<\omega_{1}$ such that $g$ " $\eta \subseteq \eta$ is also in $N_{i}$. Now, $C$ has order type $\omega_{1}$, and therefore $C \cap \delta_{N_{i}}$ has order type $\delta_{N_{i}}$ by correctness of $N_{i}$. Hence, we may find some $\eta \in \delta_{N_{i}} \cap C$ and some $\nu<\eta$ such that $[\nu, \eta]$ has empty intersection with $A_{f(\delta)}$ for every $\delta \in D$ such that $\delta \geq \delta_{N_{i}}$. But then, by definition of $g$ together with the correctness of $N_{i}$ there is some $p^{\prime}=\left(f^{\prime},\left\langle b_{\delta}^{\prime}: \delta \in D^{\prime}\right\rangle\right)$ in $N_{i} \cap E$ extending $\left(f \uparrow \delta_{N_{i}},\left\langle b_{\delta}: \delta \in D \cap \delta_{N_{i}}\right\rangle\right)$, such that $f^{\prime} \uparrow \delta_{N_{i}}=f \uparrow \delta_{N_{i}}$, and such that the least ordinal in the range of $f^{\prime}$ strictly above $\mu$ is also above $\nu$. But then $f \cup f^{\prime}$ is a function satisfying condition (1) in the definition of $\mathbb{P}_{\mathcal{A}}$ and, in 
addition, range $\left(f \cup f^{\prime}\right) \cap A_{f(\delta)}=$ range $(f) \cap A_{f(\delta)}=b_{\delta}$ for every $\delta \in D$. It then follows that $\left(f \cup f^{\prime}, \vec{b}\right)$, where $\vec{b}$ is the union of $\left\langle b_{\delta}: \delta \in D\right\rangle$ and $\left\langle b_{\delta}^{\prime}: \delta \in D^{\prime}\right\rangle$, is a condition in $\mathbb{P}_{\mathcal{A}}$ extending both $p$ and $p^{\prime}$.

Lemma 5.6 completes the proof of the proposition.

Doing minor modifications to the forcing in the proof of Proposition [5.4 it is easy to derive other similar statements from $\mathrm{PFA}^{\mathrm{fin}}\left(\omega_{1}\right)$. For example one can check that the negation of Very Weak Club Guessing (VWCG) follows from PFA ${ }^{\text {fin }}\left(\omega_{1}\right)$, where VWCG is the assertion that there is a collection $\mathcal{A}$ of size $\aleph_{1}$ consisting of subsets of $\omega_{1}$ of order type $\omega$ such that every club of $\omega_{1}$ has infinite intersection with some $A \in \mathcal{A}$. In other words, VWCG says the same thing as WCG but allowing $\aleph_{1}$-many cofinal subsets of $\delta$ for each $\delta \in \operatorname{Lim}\left(\omega_{1}\right)$. One can actually show that $\mathrm{PFA}^{\mathrm{fin}}\left(\omega_{1}\right)$ implies the negation of the even weaker versions of VWCG where one fixes a countable ordinal $\tau$ and considers sets of ordinals of order type at most $\tau$. Specifically, one has the following.

Proposition 5.7. $\mathrm{PFA}^{\text {fin }}\left(\omega_{1}\right)$ implies that for every $\tau<\omega_{1}$ and every set $\mathcal{A}$, if $\mathcal{A}$ is a collection of $\aleph_{1}-$ many sets of ordinals of order type at most $\tau$, then there is a club $C \subseteq \omega_{1}$ having finite intersection with all members of $\mathcal{A}$.

The proof of Proposition 5.7, which we will omit here, is essentially the same as the proof of Proposition 5.4

Finally we derive the failure of $\mho$ from $\operatorname{PFA}^{\mathrm{fin}}\left(\omega_{1}\right)$.

Proposition 5.8. $\mathrm{PFA}^{f i n}\left(\omega_{1}\right)$ implies $\neg \mho$.

Proof. We will prove that every instance of $\neg \mho$ follows from $\mathrm{PFA}^{\text {fin }}\left(\omega_{1}\right)$. Let $\mathcal{G}=\left\langle g_{\delta}: \delta \in \omega_{1}\right\rangle$ be such that each $g_{\delta}$ is a continuous function from $\delta$ into $\omega$ with respect to the order topology. Let $\mathbb{P}_{\mathcal{G}}$ be the forcing notion consisting of all pairs $\left(f,\left\langle d_{\xi}: \xi \in D\right\rangle\right)$ satisfying the following conditions:

(1) $f \subseteq \omega_{1} \times \omega_{1}$ is a finite strictly increasing function.

(2) For every $\xi \in \operatorname{dom}(f), \operatorname{rank}(f(\xi), f(\xi)) \geq \xi$.

(3) $D \subseteq \operatorname{dom}(f)$, and for every $\xi \in D$,

(3.1) $d_{\xi}<\omega$

(3.2) $g_{f(\xi)}$ " range $(f) \subseteq \omega \backslash\left\{d_{\xi}\right\}$, and

(3.3) $\operatorname{rank}\left(\left\{\gamma<f(\xi): g_{f(\xi)}(\gamma) \neq d_{\xi}\right\}, f(\xi)\right)=\operatorname{rank}(f(\xi), f(\xi))$.

Given conditions $p_{\epsilon}=\left(f_{\epsilon},\left\langle d_{\xi}^{\epsilon}: \xi \in D_{\epsilon}\right\rangle\right) \in \mathbb{P}_{\mathcal{G}}$ for $\epsilon \in\{0,1\}$, we say that $p_{1}$ extends $p_{0}$ if and only if $f_{0} \subseteq f_{1}, D_{0} \subseteq D_{1}$, and $d_{\xi}^{1}=d_{\xi}^{0}$ for all $\xi \in D_{0}$.

Lemma 5.9 is easy to prove by appealing to condition (2) in the definition of $\mathcal{P}_{\mathcal{G}}$, together with the openness of all $g_{\delta}^{-1}(n)$.

Lemma 5.9. For every $p=\left(f,\left\langle d_{\xi}: \xi \in D\right\rangle\right) \in \mathbb{P}_{\mathcal{G}}$ and every $\xi_{0}<\omega_{1}$ there is a condition $p^{\prime} \in \mathbb{P}_{\mathcal{G}}$ extending $p$ and such that $\xi_{0} \in \operatorname{dom}\left(f^{p^{\prime}}\right)$. Also, if $\xi \in \operatorname{dom}(f)$ is a limit ordinal and $\epsilon<f(\xi)$, then there is a condition $p^{\prime} \in \mathbb{P}_{\mathcal{G}}$ and some $\xi^{\prime}<\xi$ in $\operatorname{dom}\left(f^{p^{\prime}}\right)$ such that $f^{p^{\prime}}\left(\xi^{\prime}\right)>\epsilon$.

Proof. Let us prove the first claim (the second claim is proved similarly). We may assume that $\xi_{0} \notin \operatorname{dom}(f)$ and that $\xi_{1}=\min \left(D \backslash \xi_{0}\right)$ exists (otherwise the proof is easier). 
Note that for every $\xi^{\prime}>\xi_{1}$ in $D$ there is some $l_{\xi^{\prime}}<\omega, l_{\xi^{\prime}} \neq d_{\xi^{\prime}}$, such that $g_{f\left(\xi^{\prime}\right)}\left(f\left(\xi_{1}\right)\right)=l_{\xi^{\prime}}$. Since all $g_{f\left(\xi^{\prime}\right)}^{-1}\left(\left\{l_{\xi^{\prime}}\right\}\right)$ are open in the order topology, we may fix $\eta<f\left(\xi_{1}\right)$ such that $g_{f\left(\xi^{\prime}\right)}$ " $\left[\eta, f\left(\xi_{1}\right)\right)=\left\{l_{\xi^{\prime}}\right\}$ for every $\xi^{\prime}>\xi_{1}$ in $D$. Let $X=\left\{\gamma<f\left(\xi_{1}\right): g_{f\left(\xi_{1}\right)}(\gamma) \neq d_{\xi_{1}}\right\}$.

Since $\operatorname{rank}\left(X, f\left(\xi_{1}\right)\right)=\operatorname{rank}\left(X \backslash \eta, f\left(\xi_{1}\right)\right)=\operatorname{rank}\left(f\left(\xi_{1}\right), f\left(\xi_{1}\right)\right) \geq \xi_{1}$, we may find $\gamma \in\left[\eta, f\left(\xi_{1}\right)\right)$ such that $g_{f\left(\xi_{1}\right)}(\gamma) \neq d_{\xi_{1}}$ and such that $\operatorname{rank}(\gamma, \gamma) \geq \xi_{0}$.

Now it is easy to check that $p^{\prime}=\left(f \cup\left\{\left\langle\xi_{0}, \gamma\right\rangle\right\},\left\langle d_{\xi}: \xi \in D\right\rangle\right)$ is a condition extending $p$.

Lemma 5.10. For every $p=\left(f,\left(d_{\xi}: \xi \in D\right)\right) \in \mathbb{P}_{\mathcal{G}}$ and every $\xi \in \operatorname{dom}(f)$ there is a condition $p^{\prime} \in \mathbb{P}_{\mathcal{G}}$ extending $p$ and such that $\xi \in D^{p^{\prime}}$.

Proof. Fix two distinct colours $d, d^{\prime}$ in $\omega \backslash \operatorname{range}\left(g_{f(\xi)} \uparrow \operatorname{range}(f)\right)$. If suffices to prove that at least one of

(i) $\operatorname{rank}\left(\left\{\gamma<f(\xi): g_{f(\xi)}(\gamma) \neq d\right\}, f(\xi)\right)=\operatorname{rank}(f(\xi), f(\xi))$ and

(ii) $\operatorname{rank}\left(\left\{\gamma<f(\xi): g_{f(\xi)}(\gamma) \neq d^{\prime}\right\}, f(\xi)\right)=\operatorname{rank}(f(\xi), f(\xi))$

holds. But if $\operatorname{rank}\left(\left\{\gamma<f(\xi): g_{f(\xi)}(\gamma) \neq d\right\}, f(\xi)\right)<\operatorname{rank}(f(\xi), f(\xi))$, then $\operatorname{rank}\left(\left\{\gamma<f(\xi): g_{f(\xi)}(\gamma)=d\right\}, f(\xi)\right)=\operatorname{rank}(f(\xi), f(\xi))$ by Lemma 5.2. and therefore $\operatorname{rank}\left(\left\{\gamma<f(\xi): g_{f(\xi)}(\gamma) \neq d^{\prime}\right\}, f(\xi)\right)=\operatorname{rank}(f(\xi), f(\xi))$ since $\{\gamma<$ $\left.f(\xi): g_{f(\xi)}(\gamma)=\delta\right\}$ is contained in $\left\{\gamma<f(\xi): g_{f(\xi)}(\gamma) \neq d^{\prime}\right\}$.

It follows from the above lemmas that if $G$ is $\mathbb{P}_{\mathcal{G}}$-generic, then the union of the ranges of all first components of conditions in $G$ is a club $C$ of $\omega_{1}^{V}$ and for every $\delta \in C$ there is some $d_{\delta} \in \omega$ such that $g_{\delta}$ " $C \subseteq \omega \backslash\left\{d_{\delta}\right\}$.

Obviously, $\mathbb{P}_{\mathcal{G}}$ has cardinality $\aleph_{1}$. It remains to show the following.

Lemma 5.11. $\mathbb{P}_{\mathcal{G}}$ is finitely proper.

Proof. Let $\left\{N_{i}: i \in m\right\}$ be a finite set of countable elementary substructures of $H\left(\omega_{2}\right)$ containing $\mathbb{P}_{\mathcal{G}}$ and let $p=\left(f,\left(d_{\xi}: \xi \in D\right)\right)$ be a condition of $\mathbb{P}_{\mathcal{G}}$ such that for each $i$ :

(a) $\delta_{N_{i}}$ is a fixed point of $f$,

(b) $\delta_{N_{i}} \in D$, and

(c) $\left\{\beta<\delta_{N_{i}}: g_{\delta_{N_{i}}}(\beta) \neq d_{\delta_{N_{i}}}\right\}$ is $N_{j}$-stationary 17 for every $j \in m$ such that $\delta_{N_{i}}=\delta_{N_{j}}$.

By arguing as in the proof of Lemma [5.6 it is easy to see that such a $p$ is $\left(N_{i}, \mathbb{P}_{\mathcal{G}}\right)^{-}$ generic for all $i$. The main point is that if $C \subseteq \omega_{1}$ is a club in $N_{i}$ as in the proof of Lemma 5.6, then $C \cap\left\{\beta<\delta_{N_{i}}: g_{\delta_{N_{i}}}(\beta) \neq d_{\delta_{N_{i}}}\right\} \neq \emptyset$. But this is of course ensured by the $N_{i}$-stationarity of $\left\{\beta<\delta_{N_{i}}: g_{\delta_{N_{i}}}(\beta) \neq d_{\delta_{N_{i}}}\right\}$.

Since every condition in $\bigcap_{i<m} N_{i}$ can be extended to a condition $p=\left(f,\left(d_{\xi}: \xi \in\right.\right.$ $D)$ ) satisfying (a), the proof of the lemma will be finished once we show that every $p=\left(f,\left(d_{\xi}: \xi \in D\right)\right)$ satisfying (a) can be extended to a $\mathbb{P}_{\mathcal{G}}$-condition $p^{\prime}$ satisfying also (b) and (c). For this, let $\left(\delta_{j}\right)_{j<n}$ be the increasing enumeration of $\left\{\delta_{N_{i}}: i<\right.$ $m\}$ and let $\left(i_{k}^{j}\right)_{j<n, k<n_{j}}$ be such that $\left\{N_{i}: \delta_{N_{i}}=\delta_{j}\right\}=\left\{N_{i_{0}^{j}}, \ldots, N_{i_{n_{j}-1}^{j}}\right\}$ for all $j$. For each $j$ let $\left\{d_{0}^{j}, \ldots, d_{n_{j}}^{j}\right\}$ be such that $\left\{d_{0}^{j}, \ldots, d_{n_{j}}^{j}\right\} \cap g_{\delta_{j}} " \operatorname{range}(f)=\emptyset$.

\footnotetext{
${ }^{17}$ The concept of $M$-stationarity appears in 14. In our context, saying that $Y \subseteq \omega_{1}$ is $N$-stationary means that $Y$ intersects all clubs of $\omega_{1}$ belonging to $N$.
} 
Claim 5.12. For every $j$ there is some $d(j) \in\left\{d_{0}^{j}, \ldots, d_{n_{j}}^{j}\right\}$ such that $\left\{\beta<\delta_{N_{j}}\right.$ : $\left.g_{\delta_{N_{j}}}(\beta) \neq d(j)\right\}$ is $N_{i_{k}^{j}}$-stationary for every $k<n_{j}$.

Proof. By arguing as in the proof of Lemma 5.10 one can see that for every $k<n_{j}$ there is some $e_{k} \in\left\{d_{0}^{j}, \ldots, d_{n_{j}}^{j}\right\}$ such that $\left\{\beta<\delta_{N_{j}}: g_{d_{N_{j}}}(\beta) \neq d\right\}$ is $N_{i_{k}^{j}}$-stationary for every $d \in\left\{d_{0}^{j}, \ldots, d_{n_{j}}^{j}\right\} \backslash\left\{e_{k}\right\}$. But then, if $d \in\left\{d_{0}^{j}, \ldots, d_{n_{j}}^{j}\right\} \backslash$ $\left\{e_{0}, \ldots, e_{n_{j}-1}\right\}$, then $\left\{\beta<\delta_{N_{j}}: g_{d_{N_{j}}}(\beta) \neq d\right\}$ is $N_{i_{k}^{j}}$-stationary for every $k$.

Now we may extend $p$ to a condition $p^{\prime}$ of the forn $\bigcirc,\left(d_{\xi}^{\prime}: \xi \in D \cup\right.$ $\left.\left.\left\{\delta_{0}, \ldots, \delta_{n-1}\right\}\right)\right)$, where $d_{\xi}^{\prime}=d_{\xi}$ if $\xi \in D$ and $d_{\delta_{j}}^{\prime}=d(j) \sum_{\Perp}<n$ and $\delta_{j} \notin D$, and $p^{\prime}$ will satisfy (a)-(c). The point is that condition (3.3) in the definition of $\mathbb{P}_{\mathcal{G}}$ holds for $p^{\prime}$ thanks to (c). For this, given any $i$ and any $\nu<\delta_{N_{i}}$, let $C \in N_{i}$ be a club of $\xi<\omega_{1}$ such that $\operatorname{rank}(\xi, \xi) \geq \nu$ and note that $C \cap g_{\delta_{N_{i}}}^{-1}\left(d_{N_{i}}\right) \neq \emptyset$.

Lemma 5.11 concludes the proof of the proposition.

We do not know whether $\mathrm{PFA}^{\text {fin }}\left(\omega_{1}\right)$ implies $\neg \mho_{n}$ for any $n, 2 \leq n<\omega$. As a matter of fact, the methods in the present paper do not seem to produce models of $\neg \mho_{n}$ for any $n$. The reason is basically that if $N_{0}, \ldots, N_{m}$ are countable substructures such that $\delta=N_{0} \cap \omega_{1}=\ldots=N_{m} \cap \omega_{1}, f: \delta \longrightarrow n$, and $n \leq m$, then it need not be true that there is any $i \in m$ such that $f^{-1}(i)$ is $N_{j}$-stationary for all $j \leq m$. On the other hand, a straightworfard variation of the proof of Proposition 5.8 shows that $\neg \mho_{2}$ follows from $\operatorname{PFA}\left(\omega_{1}\right)$.

\section{ACKNOWLEDGMENTS}

The authors wish to thank Hiroshi Sakai for showing them a proof that the negation of Club Guessing is preserved by any product with finite supports of Cohen forcing and a proof that Cohen forcing adds a Weak Club Guessing sequence, and Michael Hrusak for showing them a proof that adding random reals preserves $\neg$ WCG. The authors thank two anonymous referees for urging them to isolate a toolbox of basic lemmas and write their proofs with full resolution. This has indeed made the paper more easily readable. Finally, we thank the referees for observing that Code(even-odd) follows from $\mathrm{MA}_{\omega_{1}}$ together with $\neg \mathrm{WCG} 18$

\section{REFERENCES}

[1] Uri Abraham and James Cummings, More results in polychromatic Ramsey theory, Cent. Eur. J. Math. 10 (2012), no. 3, 1004-1016, DOI 10.2478/s11533-012-0037-3. MR2902230

[2] D. Asperó and M.A. Mota, A generalization of Martin's Axiom. Preprint (2012).

[3] James E. Baumgartner, Applications of the proper forcing axiom, Handbook of set-theoretic topology, North-Holland, Amsterdam, 1984, pp. 913-959. MR776640 (86g:03084)

[4] M. Bekkali, Topics in set theory, Lecture Notes in Mathematics, vol. 1476, Springer-Verlag, Berlin, 1991. Lebesgue measurability, large cardinals, forcing axioms, rho-functions; Notes on lectures by Stevo Todorčević. MR1119303 (92m:03070)

[5] Keith J. Devlin and Hàvard Johnsbräten, The Souslin problem, Lecture Notes in Mathematics, Vol. 405, Springer-Verlag, Berlin, 1974. MR0384542 (52 \#5416)

[6] M. Foreman, M. Magidor, and S. Shelah, Martin's maximum, saturated ideals, and nonregular ultrafilters. I, Ann. of Math. (2) 127 (1988), no. 1, 1-47, DOI 10.2307/1971415. MR.924672 (89f:03043)

\footnotetext{
${ }^{18}$ In an earlier version of the paper we were focusing on Code(even-odd) rather than the stronger (and more natural) $\mathrm{MA}_{\omega_{1}}+\neg \mathrm{WCG}$.
} 
[7] D. H. Fremlin, Consequences of Martin's axiom, Cambridge Tracts in Mathematics, vol. 84, Cambridge University Press, Cambridge, 1984. MR780933 (86i:03001)

[8] Thomas Jech, Set theory, Springer Monographs in Mathematics, Springer-Verlag, Berlin, 2003. The third millennium edition, revised and expanded. MF 1940513 (2004g:03071)

[9] István Juhász, A weakening of $\mathbf{p}$, with applications to topology, Comment. Math. Univ. Carolin. 29 (1988), no. 4, 767-773. MR982796 (90d:54005)

[10] Piotr Koszmider, On strong chains of uncountable functions, Israel J. Math. 118 (2000), 289-315, DOI 10.1007/BF02803525. MR.1776085 (2001g:03091)

[11] Kenneth Kunen, Set theory, Studies in Logic and the Foundations of Mathematics, vol. 102, North-Holland Publishing Co., Amsterdam, 1980. An introduction to independence proofs. MR.597342 (82f:03001)

[12] Richard Laver, Random reals and Souslin trees, Proc. Amer. Math. Soc. 100 (1987), no. 3, 531-534, DOI 10.2307/2046443. MR891159 (88g:03068)

[13] T. Miyamoto, Club guessing on the least uncountable cardinal and $\mathrm{CH}$, unpublished (2008).

[14] Justin Tatch Moore, Set mapping reflection, J. Math. Log. 5 (2005), no. 1, 87-97, DOI 10.1142/S0219061305000407. MR2151584 (2006c:03076)

[15] Justin Tatch Moore, A five element basis for the uncountable linear orders, Ann. of Math. (2) 163 (2006), no. 2, 669-688, DOI 10.4007/annals.2006.163.669. MR2199228(2007d:03085)

[16] Justin Tatch Moore, Aronszajn lines and the club filter, J. Symbolic Logic 73 (2008), no. 3, 1029-1035, DOI 10.2178/jsl/1230396763. MR2444284(2009e:03086)

[17] Itay Neeman, Forcing with sequences of models of two types, Notre Dame J. Form. Log. 55 (2014), no. 2, 265-298, DOI 10.1215/00294527-2420666. MR.3201836

[18] J. Roitman, Adding a random or a Cohen real: topological consequences and the effect on Martin's axiom, Fund. Math. 103 (1979), no. 1, 47-60. MR.535835 (81h:03098)

[19] Saharon Shelah, Independence results, J. Symbolic Logic 45 (1980), no. 3, 563-573, DOI 10.2307/2273423. MR583374 (82b:03099)

[20] Saharon Shelah, Proper forcing, Lecture Notes in Mathematics, vol. 940, Springer-Verlag, Berlin, 1982. MR675955 (84h:03002)

[21] Saharon Shelah, Proper and improper forcing, 2nd ed., Perspectives in Mathematical Logic, Springer-Verlag, Berlin, 1998. MR1623206 (98m:03002)

[22] R. M. Solovay and S. Tennenbaum, Iterated Cohen extensions and Souslin's problem, Ann. of Math. (2) 94 (1971), 201-245. MR0294139 (45 \#3212)

[23] Stevo Todorčević, A note on the proper forcing axiom, Axiomatic set theory (Boulder, Colo., 1983), Contemp. Math., vol. 31, Amer. Math. Soc., Providence, RI, 1984, pp. 209-218, DOI 10.1090/conm/031/763902. MR763902 (86f:03089)

[24] Stevo Todorčević, Directed sets and cofinal types, Trans. Amer. Math. Soc. 290 (1985), no. 2, 711-723, DOI 10.2307/2000309. MR792822 (87a:03084)

[25] Boban Veličković, Forcing axioms and stationary sets, Adv. Math. 94 (1992), no. 2, 256-284, DOI 10.1016/0001-8708(92)90038-M. MR1174395 (93k:03045)

School of Mathematics, University of East Anglia, Norwich NR4 7TJ, United KingDOM

E-mail address: d.aspero@uea.ac.uk

Department of Mathematics, University of Toronto, Toronto, Ontario, Canada M5S $2 \mathrm{E} 4$

E-mail address: motagaytan@gmail.com

For the convenience of our readers, references now pull in directly from MathSciNet and are rendered as they appear there. When there is no MR number, the individual reference is left as it is supplied by the author. This occasionally leads to minor inconsistencies.

References that are pulled in from MathSciNet cannot be changed, and minor inconsistencies cannot be altered because of the high volume of references that appear in our papers. We thank you for your understanding. 